\title{
Nuclear-capture of endosomes drives depletion of nuclear G-actin to promote SRF/MRTF gene expression and cancer cell invasiveness
}

\section{Sergi Marco}

Beatson Institute

Matthew Neilson

Beatson Institute

Madeleine Moore

Beatson Institute

Arantxa Pérez-García

Beatson Institute for Cancer Research

Holly Hall

Beatson Institute

Louise Mitchell

Beatson Institute for Cancer Research

Giovanny Rodriguez Blanco

IGMM UoE https://orcid.org/0000-0002-7154-7244

Ann Hedley

Beatson Institute

Sara Zanivan

Beatson Institute https://orcid.org/0000-0002-9880-9099

Jim Norman ( $\nabla$ j.norman@beatson.gla.ac.uk)

CRUK-Beatson Institute

\section{Article}

Keywords: nuclear G-actin, actin polymerisation, nuclear import, EphA2, cell:cell repulsion, invasiveness, Serum-response factor (SRF), Myocardin-related transcription factor (MRTF), pancreatic adenocarcinoma (PDAC), Heptocyte growth factor (HGF), Rab17

Posted Date: August 5th, 2020

DOl: https://doi.org/10.21203/rs.3.rs-50672/v1 
License: (c) (i) This work is licensed under a Creative Commons Attribution 4.0 International License. Read Full License

Version of Record: A version of this preprint was published at Nature Communications on November 24th, 2021. See the published version at https://doi.org/10.1038/s41467-021-26839-y. 


\section{Nuclear-capture of endosomes drives depletion of nuclear G-actin to promote SRF/MRTF gene expression and cancer cell invasiveness.}

Sergi Marco ${ }^{1}$, Matthew Neilson ${ }^{1}$, Madeleine Moore ${ }^{1}$, Arantxa Perez-Garcia ${ }^{2}$, Holly Hall ${ }^{1}$, Louise Mitchell ${ }^{1}$, Giovani R. Blanco ${ }^{1}$, Ann Hedley ${ }^{1}$, Sara Zanivan ${ }^{1,2}$, Jim C. Norman ${ }^{1,2,3}$

${ }^{1}$ CRUK Beatson Institute, Glasgow G61 1BD Scotland, UK ${ }^{2}$ Institute of Cancer Sciences, University of Glasgow, Glasgow G61 1QH, Scotland, UK

${ }^{3}$ Lead contact author

Address for correspondence; j.norman@beatson.gla.ac.uk

Word count: 2433 words (including abstract, excluding references, figure legends and methods)

Short title: Nuclear-capture of endosomes drives SRF activation

Keywords: nuclear G-actin, actin polymerisation, nuclear import, EphA2, cell:cell repulsion, invasiveness, Serum-response factor (SRF), Myocardin-related transcription factor (MRTF), pancreatic adenocarcinoma (PDAC), Heptocyte growth factor (HGF), Rab17 
Signals are relayed from receptor tyrosine kinases (RTKs) at the cell surface to effector systems in the cytoplasm and nucleus, and coordination of this process is important for the execution of migratory phenotypes, such as cell scattering and invasion. The endosomal system influences how RTK signalling is coded, but the ways in which it transmits these signals to the nucleus to influence gene expression are not yet clear. Here we show that an RTK, cMET promotes Rab17dependent endocytosis of EphA2, another RTK, followed by centripetal transport of EphA2-positive endosomes. EphA2 then mediates physical capture of endosomes on the outer surface of the nucleus; a process involving interaction between the nuclear import machinery and a nuclear localisation sequence in EphA2's cytodomain. Nuclear capture of EphA2 promotes RhoGdependent phosphorylation of the actin-binding protein, cofilin to oppose nuclear import of G-actin. The resulting depletion of nuclear G-actin drives transcription of Myocardin-related transcription factor (MRTF)/serum-response factor (SRF)-target genes to implement cell scattering and the invasive behaviour of cancer cells.

Many plasma membrane receptors, including RTKs, are endocytosed following ligand engagement and trafficked through the endosomal system, and the way in which this occurs is thought to influence signalling outcomes ${ }^{1}$. An extensively studied example is the signal termination after endocytosis where ubiquitinated receptors are sorted for lysosomal degradation ${ }^{2}$. More recently, an increasingly complex picture is emerging in which certain endosomal compartments and membrane subdomains, including late and recycling endosomes, constitute platforms which influence downstream signalling coding ${ }^{3}$. Many of these endosomal compartments are positioned very close to the nucleus, and this is thought to facilitate the communication of signals to the transcriptional machinery. For example, the Rab5 effector, APPL translocates into the nucleus to drive transcription and cell proliferation, and delivery of CMET to perinuclear endosomes is necessary to promote nuclear accumulation of the STAT3 transcription factor ${ }^{4,5}$. There are numerous reports of RTKs, and the intracellular domains of RTKs, being imported into the nucleus to perform signalling roles ${ }^{6}$. However, mechanisms accounting for this have been unclear until, more recently, EGFR1 was shown to be delivered to the nucleoplasm via docking and fusion of endosomes with the nuclear membrane ${ }^{7,8}$. Indeed, these investigators provide evidence for physical association of endosomes with the nucleus as a prelude to nuclear import of an RTK and thus provide a rationale for how the endosomal system may directly interface with gene expression. These studies prompted us to investigate whether endosomes associate with the nucleus following 
activation of HGF signalling with a view to determining whether this may contribute to transcriptional landscapes which favour HGF-driven cell migratory behaviour. Here we report that activation of an RTK, cMET by HGF promotes endocytosis of EphA2, another RTK followed by capture of endosomes upon the nuclear surface, in an interaction mediated by a nuclear-localisation signal in the cytoplasmic domain of EphA2. This event promotes both actin polymerisation in the juxtanuclear region and phosphorylation of the actin monomer-binding protein, cofilin. We have then proceeded to use a combination of cell biological and mathematical modelling approaches to determine that it is the ability of nuclear-captured EphA2 to phosphorylate cofilin which leads to depletion of nuclear G-actin which, in turn, drives changes in gene expression to enable cell migration and invasion.

\section{RESULTS \& DISCUSSION}

\section{EphA2-mediates capture of endosomes at the nuclear surface}

To study the influence of cMET signalling on the intracellular destination of endosomes, we surface-labelled $\mathrm{H} 1299$ cells with a cell-impermeant biotinylation reagent, incubated the cells in the present and absence of HGF, and studied the distribution of biotinylated internalised material using fluorescence microscopy. This revealed a population of endosomes transporting plasma membrane-derived material into very close proximity of the nuclear membrane, and this population was significantly increased by HGF addition (Fig. 1a). Moreover, transmission electron microscopy (TEM) of nuclei purified from HGF-treated cells indicated endosomes containing plasma membrane-derived cargo docked with the nuclear membrane in an interaction which appears to be direct and not to rely on intervening structures, such as ER of Golgi membranes (Supplementary Fig. 1a). These observations prompted us to develop a stable isotope labelling (SILAC) proteomic approach to identify cargoes that traffic from the plasma membrane to endosomes that are physically associated with the nucleus. H1299 cells were SILAC-labelled with medium (M) or light (L) amino acids, surface-biotinylated and incubated in the presence or absence of HGF. Following this, nuclei were purified (together with their associated endosomes), biotinylated proteins isolated from these using streptavidin beads and the proteome of isolates analysed by mass spectrometry (Fig. 1b). This indicated that HGF addition promoted translocation of a number of receptors from the plasma membrane to the nucleus, and another RTK, EphA2 was prominent amongst these (Fig. 1b; supplementary spreadsheet 1). Surface biotinylation/capture-ELISA-based approaches confirmed that surface-labelled EphA2 relocated to a compartment which copurified with the nucleus within a few minutes of HGF addition, and super-resolution Airyscan microscopy confirmed that these EphA2-positive vesicles were attached to the nuclear surface 
(Fig. 1c). Furthermore, siRNA of EphA2 opposed recruitment of plasma membrane-derived vesicles to the nuclear surface indicating that EphA2 is not a passive cargo of this pathway, but that it is necessary for their delivery to and/or attachment to the nucleus (Fig. 1d). To determine how HGF drives packaging of EphA2 into endosomes, we tested the involvement of a battery of Rab GTPases and other endosomal regulators known to be involved in cell adhesion and migration (including Rabs 4, 5, 7, 11, 17, 21 and 25, CD63 and LAMP1/2) in EphA2 internalisation and trafficking. This highlighted Rab17 as being required for HGF-driven internalisation (Fig. 1e) and nuclear-capture (Fig. 1f) of EphA2, and live cell fluorescence imaging indicated that Rab17 accompanied EphA2 on its centripetal journey from the plasma membrane to vesicles captured at the nuclear surface (Fig. 1f, g, Supplementary Fig. 1c, Supplementary movie 1 ).

Nuclear location signals (NLS) are found in proteins that translocate to the nucleus and are recognised by $\alpha$-importins which recruit $\beta$-importins to promote association with the nuclear pore ${ }^{9}$. A bioinformatic analysis ${ }^{10}$ predicted a putative NLS in the juxtamembrane region of EphA2 and we generated two mutants of this (NLS1 and NLS2) designed to disrupt key basic residues responsible for $\alpha$-importin binding (Fig. 1h). Fusion of EphA2's NLS, but not NLS1 or NLS2, with GFP increased nuclear delivery of GFP, demonstrating that this sequence can mediate functional interaction with the nuclear import machinery (Supplementary Fig. 1d). Consistently, following HGF addition, EphA2 coimmunoprecipitated with importins - $\alpha 5$ and $\beta 1$ (Fig. 1i), and fluorescence time-lapse microscopy indicating that EphA2-positive vesicles associated with these components of the nuclear import machinery near the nuclear surface (Supplementary Fig. 1e). Importantly, mutation of EphA2's NLS opposed coimmunoprecipitation of EphA2 with importins (Fig. 1i) and reduced nuclear capture of EphA2 vesicles (Fig. 1j), without compromising canonical signalling downstream of EphA2 (Supplementary Fig. 1f). Taken together, these data indicate that addition of HGF promotes Rab17-dependent internalisation and centripetal trafficking of a population of endosomes which are then captured on the nuclear surface by an NLS in the cytotail of EphA2.

\section{Nuclear-capture of endosome is required for HGF-driven cell scattering and cancer cell invasion}

In the 'KPC' autochthonous mouse model of pancreatic ductal adenocarcinoma (PDAC), knockout of EphA2 opposes invasive migration and metastasis ${ }^{11}$. Invasive migration of EphA2 knockout PDAC cells towards a gradient of HGF was restored by re-expression of wild-type EphA2 and, interestingly, EphA2s with mutated NLSs are ineffective in this regard (Fig. 2a). Consistently, knockout or siRNA of EphA2 opposed scattering of H1299 and PDAC cells 
respectively and this was restored by re-expression of wild-type, but not NLS mutants of, EphA2 (Fig. 2b-d). Moreover, siRNA of Rab17 (to reduce internalisation of EphA2) opposed HGF-driven cell scattering (Fig. 2d) indicating that internalisation, centripetal transport and NLS-mediated nuclear capture of EphA2-positive endosomes is required for cells to mount a scattering response following addition of HGF.

\section{EphA2-mediated nuclear-capture influences expression of MRTF/SRF target genes}

We proposed that interaction of endosomes with the nuclear may influence invasive behaviour by controlling gene expression. We used RNAseq to profile gene expression signatures driven by HGF and to determine whether components of this are dependent on EphA2. This indicated that a number of HGF-responsive mRNAs were sensitive to siRNA of EphA2 (Fig. $2 e$; left panel). Serum-response factor (SRF) and myocardin-related transcription factor (MRTF) targets ${ }^{12,13}$ were strongly represented in this cohort of genes (Fig. 2e, right panel highlighted in red). qPCR confirmed that, in both H1299 (Fig. 2f) and KPC cells (Supplementary Fig. 2a), HGF-driven expression of four of the SRF-targets highlighted by RNAseq (ATF3, FosB, JunB and Zfp36) were restored by re-expression of wild-type EphA2, but not its NLS mutants. Rab17 knockdown opposed HGF-driven increases in ATF3, FosB, JunB and Zfp36 as well, consistent with a role for endocytosis, centripetal transport and nuclear-capture of EphA2-positive endosomes in the ability of cMET to communicate with the gene expression machinery (Supplementary Fig. 2b). Moreover, HGF-driven expression of other MRTF targets, including SRF itself and a number of cytoskeletal genes, was opposed by siRNA of EphA2 (Supplementary Fig. 2c). Chromatin immunoprecipitation ${ }^{13}$ showed $^{2}$ increased binding of MRTF (but not Elk-1) to FosB and JunB promoters following HGF addition and this was opposed by EphA2 knockdown (Fig. 2e; Supplementary Fig. 2d). Finally, cell scattering was significantly inhibited by siRNA knockdown of SRF indicating that regulation of gene expression links nuclear-capture to HGF-driven cellular responses (Supplementary Fig. 2e).

\section{Nuclear-capture influences MRTF/SRF target gene expression by depleting nuclear G- actin}

MRTF activation is controlled by G-actin availability ${ }^{14}$, so we hypothesised that nuclear capture of EphA2 may trigger changes in actin dynamics to regulate SRF/MRTF-dependent transcription. Live cell imaging indicated that HGF drove actin polymerisation in the vicinity of nuclear-captured EphA2 (Fig. 3a, top panel), and this was opposed by expression of an NLS 
mutant of EphA2 (Fig. 3a, left bottom panel). Moreover, siRNA of RhoG, an effector recruited to EphA2 ${ }^{15}$ after HGF addition (Supplementary Fig. 3a), reduced levels of F-actin in the juxtanuclear region measured in the presence of HGF (Fig. 3a, right bottom panel). Consistently, siRNA of RhoG opposed both transcription of SRF/MRTF target genes (Supplementary Fig. 3b) and HGF-driven cell scattering (Supplementary Fig. 3c). We next used DNase I in combination with fluorescence microscopy and flow cytometry to visualise G-actin, and found that addition of HGF significantly decreased nuclear, but not cytoplasmic, G-actin (Fig. 3c,dc).. Importantly, this was opposed by siRNA of EphA2 and rescued by wild-type, but not an NLS mutant of, EphA2 (Fig. 3c). These data indicate that nuclear capture of endosomes allows HGF signalling to drive depletion of nuclear (but not cytosolic) G-actin and to promote actin polymerisation in the juxtanuclear cytosol.

G-actin influences nucleocytoplasmic shuttling ${ }^{16}$ and transcriptional activity ${ }^{17}$ of MRTF/SRF, so we used high content imaging to measure nuclear shuttling of MRTF and the role that EphA2, RhoG and G-actin play in this. MRTF translocated to the nucleus following HGF addition. However, this was not opposed by knockdown of either EphA2 or RhoG (Fig. 3d). Moreover, by deploying non-polymerisable actins - actin ${ }^{\mathrm{R} 62 \mathrm{D}}$ and the nuclear targeted, NLSactin $^{\text {R62D }}$ actin $^{18}$ - we found that nuclear shuttling of MRTF was only minimally affected by levels of actin monomer in the cytoplasm and nucleus respectively (Fig. 3d). Despite this, expression of actin $^{\mathrm{R} 62 \mathrm{D}}$ profoundly inhibited MRTF-target gene expression (Supplementary Fig. 3b) and HGF-driven cell scattering (Fig. 3e). These data indicate that, although G-actin may make a contribution to nuclear translocation of MRTF, it is the levels of G-actin in the nucleus that ultimately determine its transcriptional activity in response to HGF. Consistently, when we elevated nuclear G-actin levels by knocking-down the exportin-6 transporter (XPO6) (Supplementary Fig. 3e), which mediates efflux of G-actin from the nucleus ${ }^{19}$, HGF-driven transcription of MRTF-target genes and cell scattering were inhibited (Supplementary Fig. 3f, g).

\section{Nuclear-capture promotes depletion of nuclear G-actin via phosphorylation of cofilin}

The rate of juxta-nuclear actin polymerisation (denoted by $E_{c} \mu 1$ in Fig. 4a), which we have found to be nuclear-capture and RhoG-dependent, is likely to affect availability of G-actin for nuclear import. Indeed, a recently-described pathway linking mechanical force to gene expression in the skin involves tension-induced F-actin polymerisation in the immediate vicinity of the nuclear membrane, and this restricts availability of G-actin in the nucleus to oppose transcription in a chromatin-dependent manner, but may also influence SRF/MRTF target gene expression ${ }^{20}$. Another event that might influence nucleo-cytoplasmic actin dynamics is phosphorylation of cofilin at $\operatorname{Ser}^{3}$ (by LIMK) (denoted by $E_{c} \mu 2$ in Fig. 4a) ${ }^{21}$, as this 
opposes formation of the cofilin-actin complex which is the species transported into the nucleus via importin-922. Addition of HGF increased levels of phospho-cofilin, and this was opposed by siRNA of EphA2 or RhoG and rescued by expression of wild-type (but not NLS mutants of) EphA2, indicating that cofilin phosphorylation is controlled by nuclear-capture and thus may influence communication with the transcriptional machinery. We designed a computation approach for modelling nucleo-cytoplasmic actin dynamics, and thus test the contribution of signalling events occurring downstream of EphA2 and RhoG to depletion of nuclear G-actin. This model clearly predicted that when juxta-nuclear actin polymerisation $\left(E_{c} \mu 1\right)$ and cofilin phosphorylation $\left(E_{c} \mu 2\right)(F i g .4 a)$ are both active this drives rapid depletion of nuclear G-actin and modestly increased cytosolic G-actin, thus recapitulating the actin dynamics observed experimentally following HGF addition (Fig. 4c). We then tested the consequences of independently reducing $E_{c} \mu 1$ and $E_{c} \mu 2$ to zero - i.e. mimicking selective inhibition of cytosolic actin polymerisation and cofilin phosphorylation respectively. This indicated that cofilin phosphorylation $\left(E_{c} \mu 2\right)$ was likely to exert a greater influence over the depletion of nuclear G-actin depletion than actin polymerisation ( $\left.E_{c} \mu 1\right)$ (Fig. 4c). This prediction was confirmed experimentally, as treatment with the Arp2/3 inhibitor, CK-666 ${ }^{23}$ completely opposed juxta-nuclear actin polymerisation without opposing expression of MRTFtarget genes (Supplementary Fig. 4a, b). Conversely, addition of the LIMK inhibitor, BMS-5 ${ }^{24}$ to oppose cofilin phosphorylation, completely opposed the ability of HGF to drive both expression of MRTF target genes and cell scattering (Fig. 4d, e).

To conclude, this study describes a new paradigm for transmission of signals between the plasma membrane and the nucleus in which one RTK (CMET) promotes endocytosis of another (EphA2) whose function is to capture endosomes at the nuclear surface. This nuclear captured EphA2 leads to both local actin polymerization and phosphorylation of cofilin. However, experimental observations and mathematical modelling have allowed us to conclude that it is the phosphorylation of cofilin leadingto depletion of nuclear G-actin which provides the main impetus to SRF/MRTF-dependent gene expression leading, in turn to cell scattering and invasive responses (Fig. 4e). This mechanism of signal transduction provides opportunities for coordination and integration of inputs from other pathways and therefore, we anticipate that nuclear-captured endocytic compartments will function as a key signalling nexus for a range of cellular processes in a variety of cells types. 


\section{Methods}

\section{Cell culture, transfection, constructs and siRNA}

H1299 cells were obtained from ATCC. The genetic identity of all these cell lines has been confirmed at the CRUK Beatson Institute for Cancer Research. Cell lines were cultured at 37 ${ }^{\circ} \mathrm{C}$ and $10 \% \mathrm{CO} 2$ in a humidified incubator. H1299 cells and in-house PDAC cells were cultured in DMEM. All media were supplemented with $10 \%$ foetal calf serum, $2 \mathrm{mM} \mathrm{L-}$ glutamine, $100 \mathrm{IU} / \mathrm{ml}$ penicillin, $100 \mu \mathrm{g} / \mathrm{ml}$ streptomycin and $250 \mu \mathrm{g} \mathrm{ml}{ }^{-1}$ fungizone. For expression vectors, cells were transfected using lipofectamine 2000 (Thermo Fisher), and for siRNAs transfection was performed using the Nucleofector system (kit V; Lonza).

PDAC cells were obtained from pancreatic adenocarcinoma from EphA2 ${ }^{+/+}$or $\mathrm{EphA}^{-/-} \mathrm{KPC}$

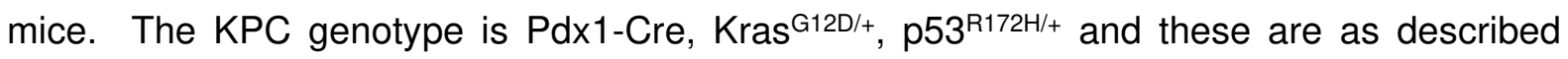
previously ${ }^{11}$. EphA2 ${ }^{-/-}$mice were obtained from Jackson laboratories.

EphA2-GFP cDNA was subcloned in a pCDNA3.1-Zeo empty vector. Mutagenesis to generate the EphA2 NLS1 and NLS2 mutants was performed using the Quikchange Multi-lightning site directed mutagenesis kit (Agilent). importin-a5 (KPNA1) and importin- $\beta 1$ (KPNB1) cDNAs were obtained from MRC PPU reagents (MRC, Dundee) and subcloned into an mCherry-N1 empty vector. mCherry-Lifeact was a gift from Laura Machesky. RhoG-GFP construct was a kind gift from Anne Ridley (University of Bristol). EGFP-C1-Rab17 was a gift from Jeremy Simpson (University College, Ireland). The NLS-R62D actin construct was a kind gift from Robert Grosse (University of Freiburg). siRNA oligos for EphA2, Rab17, SRF, RhoG and XPO6 were purchased as ON-TARGETplus siRNA SMARTpools (Dharmacon). LIMK inhibitor BMS-5/LIMKi 3 (Tocris) was used at a 10uM final concentration. Arp2/3 inhibitor CK-666 (Tocris) was used at a final concentration of $100 \mathrm{uM}$.

\section{Antibodies and immunoprecipitation}

For Western blotting and immunofluorescence, antibodies were from the following sources: goat anti-LaminA/C (Santa Cruz), mouse anti-EphA2 (Millipore), mouse anti-GFP (Immunoprecipitations, Abcam), mouse anti-GFP (Western Blot, Santa Cruz), rabbit anti-GFP (Abcam), rabbit anti-importin- $\alpha 5$ (KPNA1) (Proteintech), mouse anti-importin- $\beta 1$ (KPNB1) (Cell Signaling), mouse anti-actin (Sigma), mouse anti-RhoG (Millipore), mouse anti-Rab17 (Abnova). For immunoprecipitation, mouse antibodies were coupled to magnetic beads conjugated to anti-mouse IgG (Invitrogen; Dynabeads Sheep anti-mouse IgG; catalogue number 11031). For chromatin immunoprecipitation, antibodies were rabbit anti-SRF (Cell Signaling Technologies), rabbit anti-MRTF-A (Cell Signalling Technologies) and mouse antiELK-1 (Santa Cruz). Cell lysates were prepared in a buffer containing $200 \mathrm{mM} \mathrm{NaCl}, 75 \mathrm{mM}$ Tris- $\mathrm{HCl} \mathrm{pH} \mathrm{7,} 15 \mathrm{mM} \mathrm{NaF}, 1.5 \mathrm{mM} \mathrm{Na}_{3} \mathrm{VO}_{4}, 7.5 \mathrm{mM}$ EDTA, $7.5 \mathrm{mM}$ EGTA, 0.15\% (v/v) 
Tween-20, and protein inhibitors (Thermo Fisher). Lysates were passed three times through a 26-gauge needle and clarified by centrifugation at $10,000 \mathrm{~g}$ for $5 \mathrm{~min}$ at $4{ }^{\circ} \mathrm{C}$. Lysates were added to the beads and rotated for $2 \mathrm{~h}$ at $4{ }^{\circ} \mathrm{C}$. Beads were washed three times in Tween-20containing buffer, and then analysed by Western blotting.

\section{qRT-PCR}

Trizol (Ambion) was used to isolate total RNA from the relevant cell lines following the manufacturer protocol. The cDNA was obtained by using the Quantitect reverse transcription kit (Qiagen). qRT-PCR reactions were prepared using the SYBR Green kit (QuantaBio). The amplified products were obtained and analysed by a CFX96 qPCR System (BioRad). $\triangle \Delta C(t)$ was determined using GAPDH as a reference. Control transfected transcript levels were assigned the arbitrary value of 1 . FosB, JunB, ATF3 and GAPDH human Quantitect primers were purchased from Qiagen and the following primers manufactured by Invitrogen (Thermo Fisher):

JunB mouse

F - 5' - TCA CGA CGA CTC TTA CGC AG -3'

R - 5'- CCT TGA GAC CCC GAT AGG GA - 3'

FosB mouse

F - 5'- TTT TCC CGG AGA CTA CGA CTC -3'

R - 5' - GTG ATT GCG GTG ACC GTT G - 3'

ATF3 mouse

F - 5'-GAG GAT TTT GCT AAC CTG ACA CC -3'

R - 5' - TTG ACG GTA ACT GAC TCC AGC - 3'

Zfp36 mouse

F - 5'- CCA CCT CCT CTC GAT ACA AGA -3'

R - 5'- GCT TGG CGA AGT TCA CCC A - 3'

GAPDH mouse

F - 5' - AGG TCG GTG TGA ACG GAT TTG -3'

R - 5' - TGT AGA CCA TGT AGT TGA GGT CA - 3'

Zfp36 human

F - 5'- GAC TGA GCT ATG TCG GAC CTT -3'

R - 5'- GAG TTC CGT CTT GTA TTT GGG G - 3'

SRF human

F - 5' - ACT GCC TTC AGT AGG AAC AA - 3'

R - 5'- TTC AAG CAC ACA CAC TCA CT - 3'

ACTA2 human

F- 5' - AAA AGA CAG CTA CGT GGG TGA - 3' 
R - 5' - GCCATGTTCTATCGGGTACTTC - 3'

CFL1 human

F - 5' - TAC GCC ACC TTT GTC AAG ATG -3'

R - 5' - CCT TGG AGC TGG CAT AAA TCA T - 3'

IER5 human

F - 5' - TTT CTC GGG ACT CCT ACG GAA - 3'

R - 5' - GCT CCA GGG GTT CAT GTC TC - 3'

FOSL2 human

F - 5' - CAG AAA TTC CGG GTA GAT ATG CC - 3'

R - 5' - GGT ATG GGT TGG ACA TGG AGG - 3'

CYR61 human

F - 5' - CTC GCC TTA GTC GTC ACC C - 3'

R - 5' - CGC CGA AGT TGC ATT CCA G - 3'

MYL9 human

F - 5' - TCT TCG CAA TGT TTG ACC AGT - 3'

R - 5' - GTT GAA AGC CTC CTT AAA CTC CT - 3'

FosB promoter human

F- 5' - CCG CGA GCA GTT CCC GTC AAT CCC TC - 3'

R- 5' - GCA GTT CCT GTC TCA GAG GTC TCG TGG GC - 3'

JunB promoter human

F- 5' - CCT CCC GGG TCC CTG CAT CCC C - 3'

R- 5' - ACG CCT CTC GGC CCT CTC TTC CC - 3'

\section{Quantification of EphA2 internalisation and nuclear-capture}

Internalisation: Cell surface proteins were labelled with membrane impermeant sulfo-NHS-SSBiotin $\left(0.13 \mathrm{mg} \mathrm{ml}^{-1}\right)$ in PBS for $30 \mathrm{~min}$ at $4{ }^{\circ} \mathrm{C}$. To allow internalisation, cells were incubated at $37^{\circ} \mathrm{C}$ for the appropriate times in the presence and absence of HGF (10 ng/ml, Preprotech). To remove biotin from proteins remaining at the cell surface, cells were incubated with sodium mercaptoethanesulphonate (MesNa; $20 \mathrm{mM}$ ) for $60 \mathrm{~min}$ at $4{ }^{\circ} \mathrm{C}$, and the cell lysed in a buffer containing $1.5 \%$ triton X-100 and $0.75 \%$ NP40 (pH 7.0). Biotinylated (internalised) EphA2 was then determined by capture-ELISA. Maxisorb 96 well plates (Life Technologies) were coated overnight with $5 \mu \mathrm{g} / \mathrm{ml}$ anti-EphA2 antibodies (Millipore) in $0.05 \mathrm{M} \mathrm{Na}_{2} \mathrm{CO}_{3} \mathrm{pH} 9.6$ at $4^{\circ} \mathrm{C}$ and blocked in PBS containing $0.05 \%$ Tween-20 (PBS-T) with $5 \%$ BSA for 1 hour at room temperature. EphA2 was captured by overnight incubation of $50 \mu \mathrm{l}$ of cell lysate at $4^{\circ} \mathrm{C}$. Unbound material was removed by extensive washing with PBS-T and wells were incubated with streptavidin-conjugated horseradish peroxidase (Vector Laboratories) in PBS-T 
containing $1 \%$ BSA for $1 \mathrm{hr}$ at $4^{\circ} \mathrm{C}$. Following further washing, biotinylated integrins were detected by chromogenic reaction with ortho-phenylenediamine as described previously ${ }^{25}$.

Nuclear-capture: Cells were collected in PBS, centrifuged for $10 \mathrm{~s}$ in a table top centrifuge and the supernatant discarded. Cells were resuspended in PBS containing $0.1 \%$ NP-40 (PBS-N) and passed through a 26-gauge needle 5 times, centrifuged for 10 s and resuspended in PBS$\mathrm{N}$. This procedure was then repeated. The pellet was finally resuspended in lysis buffer containing $1 \%$ Triton X-100 and $1 \%$ NP-40, sonicated for three rounds of 20 s/round. Finally, samples were centrifuged at $8000 \mathrm{rpm}$ in a table top centrifuge for $30 \mathrm{~s}$ and protein content was measured by using Optiblot (Thermo Fisher) following the manufacturer's protocol. The levels of nuclear-captured biotinylated-EphA2 were determined by capture-ELISA as described above using ELISA plates coated with either mouse anti-EphA2 or mouse anti-GFP (Abcam) antibodies. To image purified nuclei by immunofluorescence, nuclei were seeded onto glass bottom dishes previously coated with poly-D-lysine, fixed with $4 \%$ paraformaldehyde and stained with various antibodies.

To image internalisation of biotinylated proteins, the same cell-surface biotinylation and Mes $\mathrm{Na}$ reduction procedure as described above was used followed by fixation in $4 \%$ paraformaldehyde. Biotinylated proteins were visualised with Alexa488-conjugated streptavidin (Vector). Images were acquired using a Zeiss LSM 880 Airyscan confocal microscope, and processed using Zen Black Zeiss software. To quantify distances between vesicles and the nucleus in ImageJ software a mask for the DAPI staining was created and a distance map obtained from the boundaries of the nucleus. Internalised particles were selected and the distance to the nucleus was analysed by overlaying them with the distance map.

\section{Cell scattering and invasion}

Cell scattering: $\mathrm{H} 1299$ cells were seeded onto six-well plates for $48 \mathrm{~h}$, during which time the cells formed small colonies. Cells were then visualized using a Nikon time lapse microscope in the presence and absence of HGF (10 $\mathrm{ng} \mathrm{ml}^{-1)}$, with or without CK-666 (XXX) or BMS-5 $(X X X)$. Images were collected every $5 \mathrm{~min}$ from six different regions per well. To track scattering, ImageJ manual tracking and chemotaxis plugins were used.

Invasion: Inverted Matrigel assays were performed as described previously ${ }^{26}$. Briefly, Matrigel was allowed to polymerize in Transwell inserts (Corning) for $1 \mathrm{~h}$ at $37^{\circ} \mathrm{C}$. Inserts were then inverted and cells seeded directly onto the upper face of the filter. Cells were placed in a chemotactic gradient of medium supplemented with $10 \%$ FCS and $10 \mathrm{ng} / \mathrm{ml} \mathrm{HGF}$ and, $48 \mathrm{~h}$ after seeding, migrating cells were stained with Calcein-AM and visualized by confocal microscopy with serial optical sections being captured at $15-\mu \mathrm{m}$ intervals. 


\section{High-content image analysis}

Cell scattering: PDAC cells were seeded onto glass six-well plates. After $24 \mathrm{~h}$ cells were fixed and stained with DAPI (Sigma) and Cell Mask (1:10,000, Invitrogen). Cells were imaged at 10X magnification using the Opera Phenix High-Content Screening System (PerkinElmer) and cell distribution was quantified using Columbus Image Data Storage and Analysis System (PerkinElmer). A cell cluster was defined as five or more nuclei $<1 \mu \mathrm{m}$ from each other with an area $>600 \mu \mathrm{m}^{2}$.

Nucleocytoplasmic distribution of MRTF-GFP: H1299 cells stably expressing an MRTF-GFP construct (gift from Drs. Christian Baarlink and Robert Grosse, University of Marburg) were plated in 6-well dishes and treated in the presence or absence of HGF. Cells were processed and imaged as described before. MRTF localisation was quantified by using DAPI as a nuclear mask and DAPI-negative Cell Mask staining for the cytoplasmic mask.

\section{RNAseq}

RNA was extracted as described in the qRT-PCR methods. Quality of the purified RNA was tested on a 2200 Tapestation using RNA screentape (Agilent). Libraries for cluster generation and DNA sequencing were prepared using the TruSeq Stranded mRNA LT Kit (Illuma). Quality and quantity of the DNA libraries was assessed on the 2200 Tapestation (D1000 screentape) and Qubit (Thermo Fisher Scientific) respectively. The libraries were run on the Next Seq 500 (Illumina) using the High Output 75 cycles kit (2x36cycles, paired end reads, single index). To analyse the RNAseq expression data quality checks on the raw RNASeq data files were performed using fastqc version 0.11 .7 and fastq screen version 0.12.0. RNAseq paired-end reads were aligned to the $\mathrm{GRCm} 38$ version of the human genome and annotation using HiSat2 version 2.1.0. Expression levels were determined and statistically analysed using a combination of HTSeq version 0.9.1, the R environment version 3.4.4, utilizing packages from the Bioconductor data analysis suite and differential gene expression analysis was performed using voom pipeline from the limma package in $\mathrm{R}$.

\section{Chromatin immunoprecipitation}

H1299 cells were incubated in the presence or absence of HGF and cross-linked by adding paraformaldehyde (final concentration 1\%) for 10min at room temperature. Glycine (125 mM) was added and incubated for further $5 \mathrm{~min}$. Harvested cells were spun and washed twice in PBS and once in PBS/NP40. Pellets were incubated for $30 \mathrm{~min}$ in ice-cold high-salt buffer, and hypotonic disruption was then performed by incubating the pellets in low-salt buffer for 30min. Samples were mechanically disrupted with a 26G syringe, centrifuged and resuspended in low-salt buffer and $20 \%$ sarkosyl. DNA pellets were obtained by centrifuging the samples on a sucrose cushion. Sonication was performed in a 30s-on 30s-off fashion for 
30min at $4{ }^{\circ} \mathrm{C}$ and the resulting DNA was quantified using Qubit ds HS DNA kit (Invitrogen). $10 \%$ of each sample was kept as inputs and $25 \mu \mathrm{g}$ of DNA were used for immunoprecipitation using antibodies recognising either MRTF, SRF or Elk-1 ( $5 \mu \mathrm{g}$ per sample) and magnetic beads (MagnaBind Goat anti-rabbit IgG or Dynabeads Sheep anti-mouse - Thermo Scientific). The immunoprecipitation was performed overnight at $4 \stackrel{\circ}{\circ}$ and samples were washed in RIPA buffer containing $\mathrm{LiCl}$ buffer and then in a Tris-EDTA buffer. . Eluted samples were treated with Proteinase $\mathrm{K}$ and cleaned using a PCR clean up kit (Qiagen). The final eluates were quantified by qPCR.

\section{Proteomics}

SILAC labelling and isolation of cell surface and nuclear proteomes: To obtain the surface proteome, H1299 cell were SILAC-labelled with heavy (Cambridge Isotope Laboratories) and light (Sigma) amino acids. SILAC-labelled cells were plated into $15 \mathrm{~cm}$ plastic dishes and, 48 $\mathrm{h}$ later, were surface-biotinylated using sulpho-NHS-SS-Biotin $(0.15 \mathrm{mg} / \mathrm{ml})$ in PBS at $4^{\circ} \mathrm{C}$ for $1 \mathrm{~h}$. Heavy-labelled cells were incubated with MesNa in Tris-buffered saline ( $\mathrm{pH} \mathrm{8.6)} \mathrm{for} 50$ $\min$ at $4^{\circ} \mathrm{C}$ to remove biotin from the cell surface, whilst the light-labelled cells remained unreduced. Cells were lysed and lysates from the heavy and light SILAC-labelled cells were mixed, and biotinylated proteins were captured by incubation with streptavidin-agarose beads (Upstate, Millipore) for $1.5 \mathrm{~h}$ at $4^{\circ} \mathrm{C}$ with constant rotation. Following extensive washing, biotinylated proteins were eluted from the beads by reduction with dithiotheitol $(0.1 \mathrm{M})$ in a Tris buffer ( $\mathrm{pH} \mathrm{7.5).} \mathrm{To} \mathrm{determine} \mathrm{the} \mathrm{proteins} \mathrm{which} \mathrm{translocate} \mathrm{from} \mathrm{the} \mathrm{cell} \mathrm{surface} \mathrm{to} \mathrm{the}$ nucleus, cells were labelled with light or heavy SILAC amino acids and surface biotinylated. Light-labelled cells were warmed to $37^{\circ} \mathrm{C}$ for $30 \mathrm{~min}$ to allow internalisation of labelled protein in the presence of HGF $(10 \mathrm{ng} / \mathrm{ml})$, whilst the heavy labelled cells were warmed to $37^{\circ} \mathrm{C}$ in the absence of HGF. Biotin remaining at the cell surface was removed by surface reduction as above. Cells were washed in PBS and nuclei were purified as described above. The supernatants from the heavy and light SILAC labelled cells were then mixed, and biotinylated proteins isolated using streptavidin-conjugated agarose beads as described above.

Mass spectrometry: Proteins were separated on 4-12\% gradient NuPAGE Novex Bis-Tris gel (Life Technologies) and visualised using Instant blue (Expedeon). Each gel lane was excised into 6 slices which were reduced using $10 \mathrm{mM}$ dithiothreitol, alkylated with $55 \mathrm{mM}$ iodoacetamide, and digested with trypsin (Trypsin gold, Promega), overnight at $35^{\circ} \mathrm{C}$. Tryptic peptides were desalted and dried in a centrifugal evaporator. Dried tryptic peptides were resuspended in and loaded with a buffer containing $2 \%$ acetonitrile and $0.1 \%$ formic acid and separated a $20 \mathrm{~cm}$ fused silica emitter (New Objective) packed in house with reverse phase

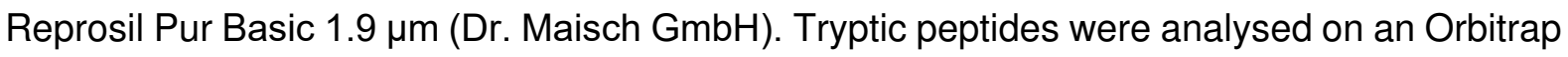
Q-Exactive HF mass spectrometer (Thermo Fisher Scientific) coupled online to an EASY-nLC 
II (Thermo Fisher Scientific). For the full scan a resolution of 60,000 at 250 Th was used. The top ten most intense ions in the full MS were isolated for fragmentation with a target of 50,000 ions at a resolution of 15,000 at 250 Th. MS data were acquired using the XCalibur software (Thermo Fisher Scientific).

Analysis of mass spectrometry data: Mass spectrometry files were processed with MaxQuant software ${ }^{27}$ version 1.5.5.1, and searched with the Andromeda search engine ${ }^{28}$ against the human UniProt database (09/07/2016; 92,939 entries). Common reverse and contaminant hits (as defined in MaxQuant output) were removed. Only protein groups identified with at least one uniquely assigned peptide were used for the analysis. For the SILAC experiments, the SILAC ratios between light-isotope and medium-isotope peptides were calculated using MaxQuant. Protein groups were considered reproducibly quantified if identified and quantified in both the surface proteome and the nuclear-capture proteome

\section{Live imaging microscopy}

Cells expressing EphA2-GFPs in combination with either mCherry-Lifeact or Rab17-mCherry and were cultured on glass-bottom $35 \mathrm{~mm}$ dishes. To obtain time lapse movies the cells were imaged in a Zeiss LSM 880 Airyscan confocal microscope at $37^{\circ} \mathrm{C}$ with controlled humidity and $\mathrm{CO}_{2}$. The first images were obtained in the absence of HGF, and then cells were treated with HGF (10 ng/ml) and imaged at time intervals of $1 \mathrm{~min}$ for $40 \mathrm{~min}$. Raw images were processed and subjected to deconvolution using Zen Black Zeiss software.

For quantification of actin polymerisation, deconvolved images were analysed in ImageJ software by measuring the mean intensity value for the mCherry-Lifeact signal in each time point in a region of interest that comprised the perinuclear internalised EphA2-GFP-positive vesicles. F-actin in the perinuclear region was then expressed as a ratio of the mean intensity value of the perinuclear region versus the mean intensity value in the cortical actin region.

\section{Transmission electron microscopy}

Purified nuclei were fixed for 2 hours in and ice-cold buffer containing $2.5 \%$ glutaraldehyde, $4 \%$ paraformaldehyde and $0.1 \mathrm{M}$ sodium cacodylate $\mathrm{pH} 7.4$. After fixation, nuclei were rinsed with a buffer containing $0.1 \mathrm{M}$ sodium cacodylate and $2 \%$ sucrose. To visualise internalised biotin, streptavidin-conjugated nanogold beads (10nm InnovaCoat $\AA_{\text {GOLD } 100 D}$ Streptavidin Conjugate - Expedeon) were used to stain the samples. Resin embedding, ultra-thin sectioning and staining for transmission electron microscopy were performed by the Electron Microscopy service (University of Glasgow). Images were obtained using a JEOL 1200 TEM - 80kv electron microscope. 


\section{Quantification of nuclear G-actin}

Purified nuclei were resuspended in PBS, fixed in $4 \%$ paraformaldehyde for $20 \mathrm{~min}$ and permeabilised with $0.2 \%$ Triton X-100 in PBS for 10 min at $37^{\circ} \mathrm{C}$. Nuclei were stained with Alexa594-conjugated DNAsel $(0.3 \mu \mathrm{M})$ in combination with DAPI for $1 \mathrm{~h} 30 \mathrm{~min}$ at $37^{\circ} \mathrm{C}$ under continuous shaking, followed by resuspension in PBS containing $1 \mathrm{mM}$ EDTA and $1 \mathrm{mM} \mathrm{MgCl}$. Data were acquired in LSRFortessa flow cytometer and analysed with the FlowJo software. Geometrical mean value corresponding to the DAPI positive population was calculated, and data were expressed as the ratio of the geometrical mean value of HGF treated cells versus vehicle treated cells.

\section{Computational model to predict nuclear G-actin dynamics}

We model the cell as two static circular compartments, $\Omega_{c}$ and $\Omega_{n}$, which represent the cytoplasm and the nucleus, respectively,

$$
\begin{aligned}
& \Omega_{C}=\left\{\boldsymbol{x} \in \mathbb{R}^{2}:\left\|\boldsymbol{x}-\boldsymbol{p}_{1}\right\|>r_{1} \cap\left\|\boldsymbol{x}-\boldsymbol{p}_{2}\right\|>r_{2}\right\}, \\
& \Omega_{n}=\left\{\boldsymbol{x} \in \mathbb{R}^{2}:\left\|\boldsymbol{x}-\boldsymbol{p}_{1}\right\|<r_{1}\right\},
\end{aligned}
$$

for some points $\boldsymbol{p}_{1}$ and $\boldsymbol{p}_{2}$, and some radii $r_{1}$ and $r_{2}$. We also let $\Gamma_{c}$ and $\Gamma_{n}$ denote the external boundary of the cytoplasm and the nucleus:cytoplasm boundary respectively,

$$
\begin{aligned}
& \Gamma_{c}=\left\{\boldsymbol{x} \in \mathbb{R}^{2}:\left\|\boldsymbol{x}-\boldsymbol{p}_{2}\right\|=r_{2}\right\}, \\
& \Gamma_{n}=\left\{\boldsymbol{x} \in \mathbb{R}^{2}:\left\|\boldsymbol{x}-\boldsymbol{p}_{1}\right\|=r_{1}\right\} .
\end{aligned}
$$

A graphical illustration of the simulation domain is provided (Supplementary Fig. 5).

It is assumed that the cytoplasm contains a population of G-actin, $G_{c}(\boldsymbol{x}, t)$, which diffuses and polymerises to form F-actin, $F_{c}(\boldsymbol{x}, t)$, at a rate of $\gamma_{G_{c}}$. In the presence of a catalytic growth factor, $E_{c}(\boldsymbol{x}, t), G_{c}(\boldsymbol{x}, t)$ polymerises to form $F_{c}(\boldsymbol{x}, t)$ at a rate of $\gamma_{G_{c}}\left(1+\mu_{1} E_{c}\right) . F_{c}(\boldsymbol{x}, t)$ depolymerises at a rate of $\gamma_{F_{c}}$ to form $G_{c}(\boldsymbol{x}, t)$. We also assume that the cytoplasm contains a population of cofilin, $C_{c}(\boldsymbol{x}, t)$, which associates with $G_{c}(\boldsymbol{x}, t)$ at a rate of $\alpha_{\Xi_{c}}$ to form cofil-actin, $\Xi_{c}(\boldsymbol{x}, t)$, and dissociates from $\Xi_{c}(\boldsymbol{x}, t)$ at a rate of $\gamma_{\Xi_{c}}$. Additionally, $C_{c}(\boldsymbol{x}, t)$ is assumed to phosphorylate at a rate of $\alpha_{\Theta_{c}}\left(1+\mu_{2} E_{c}\right)$, giving phospho-cofilin, $\Theta_{c}(\boldsymbol{x}, t)$, which dephosphorylates at a rate of $\gamma_{\Theta_{c}}$. Finally, we assume that the cytoplasm contains a population of profilin, $P_{c}(\boldsymbol{x}, t)$, which associates with $G_{c}(\boldsymbol{x}, t)$ at a rate of 
$\alpha_{\Upsilon_{c}}$ to form profil-actin, $\Upsilon_{c}(\boldsymbol{x}, t)$, and dissociates from $\Upsilon_{c}(\boldsymbol{x}, t)$ at a rate of $\gamma_{\Upsilon_{c}}$. The governing equations in the cytoplasm are therefore

$$
\begin{aligned}
& \frac{\partial G_{C}}{\partial t}=D_{G_{c}} \nabla^{2} G_{c}+\gamma_{F_{c}} F_{c}-\gamma_{G_{c}}\left(1+\mu_{1} E_{c}\right) G_{c}+\gamma_{\Xi_{c}} \Xi_{c}-\alpha_{\Xi_{c}} G_{c} C_{c}+\gamma_{\Upsilon_{c}} \Upsilon_{c}-\alpha_{\Upsilon_{c}} G_{c} P_{c}, \\
& \frac{\partial F_{c}}{\partial t}=D_{F_{c}} \nabla^{2} F_{c}-\gamma_{F_{c}} F_{c}+\gamma_{G_{c}}\left(1+\mu_{1} E_{c}\right) G_{c} \\
& \frac{\partial C_{c}}{\partial t}=D_{C_{c}} \nabla^{2} C_{c}-\alpha_{\Xi_{c}} G_{c} C_{c}+\gamma_{\Xi_{c}} \Xi_{c}-\alpha_{\Theta_{c}}\left(1+\mu_{2} E_{c}\right) C_{c}+\gamma_{\Theta_{c}} \Theta_{c}, \\
& \frac{\partial \Xi_{c}}{\partial t}=D_{\Xi_{c}} \nabla^{2} \Xi_{c}-\gamma_{\Xi_{c}} \Xi_{c}+\alpha_{\Xi_{c}} G_{c} C_{c} \\
& \frac{\partial \mathrm{P}_{c}}{\partial t}=D_{\mathrm{P}_{c}} \nabla^{2} \mathrm{P}_{c}-\alpha_{\Upsilon_{c}} G_{c} P_{c}+\gamma_{\Upsilon_{c}} \Upsilon_{c} \\
& \frac{\partial \Upsilon_{c}}{\partial t}=D_{\Upsilon_{c}} \nabla^{2} \Upsilon_{c}-\gamma_{\Upsilon_{c}} \Upsilon_{c}+\alpha_{\Upsilon_{c}} G_{c} P_{c} \\
& \frac{\partial \Theta_{c}}{\partial t}=D_{\Theta_{c}} \nabla^{2} \Theta_{c}-\gamma_{\Theta_{c}} \Theta_{c}+\alpha_{\Theta_{c}}\left(1+\mu_{2} E_{c}\right) C_{c}, \\
& \frac{\partial \mathrm{E}_{c}}{\partial t}=D_{\mathrm{E}_{c}} \nabla^{2} \mathrm{E}_{c}-\gamma_{\mathrm{E}_{c}}
\end{aligned}
$$

for $\boldsymbol{x} \in \Omega_{c}$, where $D_{[\cdot]}$ denotes the diffusion coefficient of species [·].

In the nucleus, we assume that there exists a population of nuclear G-actin, $G_{n}(x, t)$, and a population of cofilin, $C_{n}(\boldsymbol{x}, t)$, which associate with each other at a rate of $\alpha_{\Xi_{n}}$ to form nuclear cofil-actin, $\Xi_{n}(\boldsymbol{x}, t)$. Nuclear cofil-actin dissociates at a rate of $\gamma_{\Xi_{n}}$ to give $G_{n}(\boldsymbol{x}, t)$ and $C_{n}(\boldsymbol{x}, t)$. Finally, we assume that the nucleus contains a population of profilin, $P_{n}(\boldsymbol{x}, t)$, which associates with $G_{n}(\boldsymbol{x}, t)$ at a rate of $\alpha_{\Upsilon_{n}}$ to form nuclear profil-actin, $\Upsilon_{n}(\boldsymbol{x}, t)$, and dissociates from $\Upsilon_{n}(\boldsymbol{x}, t)$ at a rate of $\gamma_{\Upsilon_{n}}$. The governing equations in the nucleus are therefore

$$
\begin{aligned}
& \frac{\partial G_{n}}{\partial t}=D_{G_{n}} \nabla^{2} G_{n}+\gamma_{\Xi_{n}} \Xi_{n}-\alpha_{\Xi_{n}} G_{n} C_{n}+\gamma_{\Upsilon_{n}} \Upsilon_{n}-\alpha_{\Upsilon_{n}} G_{n} P_{n}, \\
& \frac{\partial C_{n}}{\partial t}=D_{C_{n}} \nabla^{2} C_{n}-\alpha_{\Xi_{n}} G_{n} C_{n}+\gamma_{\Xi_{n}} \Xi_{n}, \\
& \frac{\partial \Xi_{n}}{\partial t}=D_{\Xi_{n}} \nabla^{2} \Xi_{n}-\gamma_{\Xi_{n}} \Xi_{n}+\alpha_{\Xi_{n}} G_{n} C_{n}, \\
& \frac{\partial \mathrm{P}_{n}}{\partial t}=D_{\mathrm{P}_{n}} \nabla^{2} \mathrm{P}_{n}-\alpha_{\Upsilon_{n}} G_{n} P_{n}+\gamma_{\Upsilon_{n}} \Upsilon_{n}, \\
& \frac{\partial \Upsilon_{n}}{\partial t}=D_{\Upsilon_{n}} \nabla^{2} \Upsilon_{n}-\gamma_{\Upsilon_{n}} \Upsilon_{n}+\alpha_{\Upsilon_{n}} G_{n} P_{n},
\end{aligned}
$$

for $x \in \Omega_{n}$.

At the external boundary of the cytoplasm, each species obeys the no-flux condition 


$$
\frac{\partial G_{c}}{\partial \boldsymbol{n}}=\frac{\partial F_{c}}{\partial \boldsymbol{n}}=\frac{\partial C_{c}}{\partial \boldsymbol{n}}=\frac{\partial \Xi_{c}}{\partial \boldsymbol{n}}=\frac{\partial P_{c}}{\partial \boldsymbol{n}}=\frac{\partial \Upsilon_{c}}{\partial \boldsymbol{n}}=\frac{\partial \Theta_{c}}{\partial \boldsymbol{n}}=\frac{\partial \mathrm{E}_{c}}{\partial \boldsymbol{n}}=0, \quad \boldsymbol{x} \in \Gamma_{c},
$$

where $\boldsymbol{n}$ denotes the outwards-facing unit normal vector. At the internal boundary between the cytoplasm and the nucleus, we impose the boundary conditions

$$
\begin{aligned}
D_{P_{c}} \frac{\partial P_{c}}{\partial \boldsymbol{n}} & =\beta_{P_{n}} P_{n}-\beta_{P_{c}} P_{c}, \\
-D_{P_{n}} \frac{\partial P_{n}}{\partial \boldsymbol{n}} & =\beta_{P_{c}} P_{c}-\beta_{P_{n}} P_{n}, \\
D_{\Xi_{c}} \frac{\partial \Xi_{c}}{\partial \boldsymbol{n}} & =-\beta_{\Xi_{c}} \Xi_{c}, \\
-D_{\Xi_{n}} \frac{\partial \Xi_{n}}{\partial \boldsymbol{n}} & =\beta_{\Xi_{c}} \Xi_{c}, \\
D_{\mathrm{C}_{c}} & \frac{\partial \mathrm{C}_{c}}{\partial \boldsymbol{n}}=\beta_{\mathrm{C}_{n}} \mathrm{C}_{n}-\beta_{\mathrm{C}_{c}} \mathrm{C}_{c}, \\
-D_{\mathrm{C}_{n}} \frac{\partial \mathrm{C}_{n}}{\partial \boldsymbol{n}} & =\beta_{\mathrm{C}_{c}} \mathrm{C}_{c}-\beta_{\mathrm{C}_{n}} \mathrm{C}_{n}, \\
D_{\Upsilon_{c}} \frac{\partial \Upsilon_{c}}{\partial \boldsymbol{n}} & =\beta_{\Upsilon_{n}} \Upsilon_{n}, \\
-D_{\Upsilon_{n}} \frac{\partial \Upsilon_{n}}{\partial \boldsymbol{n}} & =-\beta_{\Upsilon_{n}} \Upsilon_{n},
\end{aligned}
$$

for $x \in \Gamma_{n}$, where $\beta_{[\cdot]}$ denotes the transfer rate between the cytoplasm and the nucleus for species [·]. The remaining nuclear boundary conditions are taken to be

$$
\frac{\partial G_{c}}{\partial \boldsymbol{n}}=\frac{\partial G_{n}}{\partial \boldsymbol{n}}=\frac{\partial F_{c}}{\partial \boldsymbol{n}}=\frac{\partial \Theta_{c}}{\partial \boldsymbol{n}}=\frac{\partial \mathrm{E}_{c}}{\partial \boldsymbol{n}}=0, \quad \boldsymbol{x} \in \Gamma_{n} .
$$

Simulations were performed in the Virtual Cell modelling and simulation software environment ${ }^{29}$, using the built-in Semi-Implicit Finite Volume-Particle Hybrid (regular grid, fixed time step) solver. The domain, defined by equations (1)-(4), was approximated using a rectangular Cartesian mesh comprising $201 \times 201$ grid points, and all simulations used a time-step size of 0.01 time units.

Default simulation parameters are as follows:

\section{Quantity}

Centre of the nucleus

Centre of the cell

\section{Symbol Value}

$\boldsymbol{p}_{1}$

$(0,-0.35)$

$p_{2}$ 
Centre of the catalytic growth factor

$$
p_{3}
$$

Radius of the nucleus

$r_{1}$

$5 \times 10^{-1}$

Radius of the cell

Radius of the catalytic growth factor

$r_{2} 1$

Diffusion coefficient of G-actin in the cytoplasm

Diffusion coefficient of F-actin in the cytoplasm

Diffusion coefficient of cofilin in the cytoplasm

Diffusion coefficient of cofilin-actin in the cytoplasm

Diffusion coefficient of profilin in the cytoplasm

Diffusion coefficient of profilin-actin in the cytoplasm

Diffusion coefficient of phospho-cofilin in the cytoplasm

Diffusion coefficient of catalytic growth factor in the cytoplasm

Diffusion coefficient of G-actin in the nucleus

$r_{3} \quad 3 \times 10^{-1}$

$D_{G_{c}} \quad 1 \times 10^{-1}$

$D_{F_{C}} \quad 0$

$D_{C_{c}} \quad 1 \times 10^{-1}$

$D_{\Xi_{c}} \quad 1 \times 10^{-1}$

$D_{P_{c}} \quad 1 \times 10^{-1}$

$D_{\Upsilon_{c}} \quad 1 \times 10^{-1}$

$D_{\Theta_{c}} \quad 1 \times 10^{-1}$

$D_{E_{c}} \quad 0$

$D_{G_{n}} \quad 1 \times 10^{1}$

Diffusion coefficient of cofilin in the nucleus

Diffusion coefficient of cofilin-actin in the nucleus

Diffusion coefficient of profilin in the nucleus

Diffusion coefficient of profilin-actin in the nucleus

Rate of polymerisation for G-actin in the cytoplasm

Rate of depolymerisation for F-actin in the cytoplasm

Catalytic rate parameter for actin polymerisation

Rate of association between G-actin and cofilin in the cytoplasm

Rate of dissociation for cofilin-actin in the cytoplasm

Rate of association between G-actin and profilin in the cytoplasm

Rate of dissociation for profilin-actin in the cytoplasm

Rate of phosphorylation for cofilin in the cytoplasm

Rate of dephosphorylation for phospho-cofilin in the cytoplasm

Catalytic rate parameter for cofilin phosphorylation

Decay rate of catalytic growth factor

Rate of association between G-actin and cofilin in the nucleus

Rate of dissociation for cofilin-actin in the nucleus

Rate of association between G-actin and profilin in the nucleus

$D_{C_{n}} \quad 1 \times 10^{1}$

$D_{\Xi_{n}} \quad 1 \times 10^{1}$

$D_{P_{n}} \quad 1 \times 10^{1}$

$D_{\Upsilon_{n}} \quad 1 \times 10^{1}$

$\gamma_{G_{c}} \quad 2 \times 10^{-2}$

$\gamma_{F_{C}} \quad 1 \times 10^{-2}$

$\mu_{1} \quad 1$

$\alpha_{\Xi_{c}} \quad 1 \times 10^{-1}$

$\gamma_{\Xi_{c}} \quad 1 \times 10^{-2}$

$\alpha_{\Upsilon_{c}} \quad 1 \times 10^{-2}$

$\gamma_{\Upsilon_{c}} \quad 1$

$\alpha_{\Theta_{c}} \quad 2 \times 10^{-2}$

$\gamma_{\Theta_{c}} \quad 4 \times 10^{-2}$

$\mu_{2} \quad 5 \times 10^{1}$

$\gamma_{E_{c}} \quad 0$

$\alpha_{\Xi_{n}} \quad 1 \times 10^{-2}$

$\gamma_{\Xi_{n}} \quad 1$

$\alpha_{\Upsilon_{n}} \quad 1 \times 10^{-1}$ 
Rate of dissociation for profilin-actin in the nucleus

Nuclear import rate of profilin

Nuclear export rate of profilin

Nuclear export rate of profilin-actin

Nuclear import rate of cofilin

Nuclear export rate of cofilin

Nuclear import rate of cofilin-actin

$\begin{array}{ll}\gamma_{\Upsilon_{n}} & 1 \times 10^{-2} \\ \beta_{P_{c}} & 1 \\ \beta_{P_{n}} & 1 \\ \beta_{\Upsilon_{n}} & 1 \\ \beta_{C_{c}} & 1 \\ \beta_{C_{n}} & 1 \\ \beta_{\Xi_{c}} & 2\end{array}$

It was assumed that F-actin and the catalytic growth factor are both non-diffusing, such that $D_{F_{c}}=D_{E_{c}}=0$. The remaining diffusion coefficients were chosen such that $D_{[\cdot]_{n}} \gg D_{[\cdot]_{c}}$ to ensure that the concentration profile of each species remains reasonably 'flat' within the nucleus (relative to the cytoplasm). We also made the assumption that, once introduced, the total amount catalytic growth factor is constant over time, such that $\gamma_{E_{c}}=0$. In the model, the primary purpose of cofilin is to transport G-actin from the cytoplasm to the nucleus; we therefore set $\alpha_{\Xi_{c}}>\alpha_{\Xi_{n}}$ (i.e. cofilin and G-actin associate at a faster rate in the cytoplasm than in the nucleus) and $\gamma_{\Xi_{c}}<\gamma_{\Xi_{n}}$ (i.e. cofilin-actin disassociates at a faster rate in the nucleus than in the cytoplasm), so that cofilin-actin quickly dissociates upon entering the nucleus. Since the profilin species is used to transport G-actin from the nucleus to the cytoplasm, we set $\alpha_{Y_{n}}>$ $\alpha_{\gamma_{c}}$ (i.e. profilin and G-actin associate at a faster rate in the nucleus than in the cytoplasm) and $\gamma_{\Upsilon_{n}}<\gamma_{Y_{c}}$ (i.e. profil-actin disassociates at a faster rate in the cytoplasm than in the nucleus), so that profilin-actin quickly dissociates upon entering the cytoplasm. To ensure that the equilibrium concentration of cofilin is approximately the same on either side of the nuclear boundary, we set $\beta_{C_{c}}=\beta_{C_{n}}$; similarly, we set $\beta_{P_{c}}=\beta_{P_{n}}$ so that the equilibrium concentration of profilin is approximately the same on either side of the nuclear boundary. Wet lab experiments have revealed that, in the absence of a catalytic growth factor, the concentration of G-actin in the nucleus is typically higher than the concentration of G-actin in the cytoplasm; given sufficiently large values for $\gamma_{\Xi_{n}}$ and $\gamma_{Y_{c}}$, this implies that $\beta_{\Xi_{c}}>\beta_{Y_{n}}$. Finally, the first catalytic rate parameter, $\mu_{1}$, was chosen to ensure that G-actin is polymerised at a faster rate in the presence of a catalytic growth factor (as observed experimentally), whilst the second catalytic rate parameter, $\mu_{2}$, was chosen to ensure that cofilin is rapidly phosphorylated in the presence of a catalytic growth factor (which should have the effect of reducing the amount of cofilin that is available to transport G-actin from the cytoplasm to the nucleus). 
To determine appropriate initial conditions, the model system was simulated using the default parameters outlined above (with $E_{c}(\boldsymbol{x}, t)=0, \forall \boldsymbol{x} \in \Omega_{c}$ ) until it had approached equilibrium, and then the average concentration of each species (across its associated domain) was used as a spatially constant initial value in subsequent simulations. The computed initial value for each species is as follows:

\section{Symbol Value}

\section{Quantity}

Initial concentration of G-actin in the cytoplasm

$G_{C_{\text {init }}} \quad 2.64 \times 10^{-1}$

Initial concentration of F-actin in the cytoplasm

$F_{C_{\text {init }}} \quad 5.28 \times 10^{-1}$

Initial concentration of catalytic growth factor

$E_{c_{\text {init }}} \quad 10$

Initial concentration of cofilin in the cytoplasm

$C_{c_{\text {init }}}$

$1.40 \times 10^{-1}$

Initial concentration of cofilin-actin in the cytoplasm

$\Xi_{c_{\text {init }}} \quad 9.12 \times 10^{-3}$

Initial concentration of profilin in the cytoplasm

Initial concentration of profilin-actin in the cytoplasm

$P_{c_{\text {init }}}$

$1.34 \times 10^{-1}$

Initial concentration of phospho-cofilin in the cytoplasm

$\Upsilon_{c_{\text {init }}}$

$3.95 \times 10^{-3}$

Initial concentration of G-actin in the nucleus

$\Theta_{c_{\text {init }}}$

$6.99 \times 10^{-2}$

Initial concentration of cofilin in the nucleus

$G_{n_{\text {init }}}$

$8.56 \times 10^{-1}$

Initial concentration of cofilin-actin in the nucleus

$C_{n_{\text {init }}}$

$1.50 \times 10^{-1}$

Initial concentration of profilin in the nucleus

$\Xi_{n_{\text {init }}}$

$1.21 \times 10^{-2}$

Initial concentration of profilin-actin in the nucleus

$\begin{array}{ll}P_{n_{\text {init }}} & 1.27 \times 10^{-1} \\ Y_{n_{\text {init }}} & 2.74 \times 10^{-3}\end{array}$

Note that the equilibrium concentration profile for each species is not necessarily flat, so our simulations (which use a spatially constant value as the initial condition for each species) do not start at steady state. Finally, the initial condition for the catalytic growth factor was chosen to be

$$
E(\boldsymbol{x}, 0)=\left\{\begin{aligned}
E_{\mathrm{cinit}^{\prime}}, & \left\|\boldsymbol{x}-\boldsymbol{p}_{3}\right\|<r_{3}, \quad \boldsymbol{x} \in \Omega_{c} \\
0, & \text { otherwise }
\end{aligned}\right.
$$




\section{References}

1 Platta, H. W. \& Stenmark, H. Endocytosis and signaling. Curr Opin Cell Biol 23, 393-403, doi:10.1016/j.ceb.2011.03.008 (2011).

2 Haglund, K. et al. Multiple monoubiquitination of RTKs is sufficient for their endocytosis and degradation. Nat Cell Biol 5, 461-466, doi:10.1038/ncb983 (2003).

3 Villasenor, R., Kalaidzidis, Y. \& Zerial, M. Signal processing by the endosomal system. Curr Opin Cell Biol 39, 53-60, doi:10.1016/j.ceb.2016.02.002 (2016).

4 Benmerah, A. Endocytosis: signaling from endocytic membranes to the nucleus. Curr Biol 14, R314-316, doi:10.1016/j.cub.2004.03.053 (2004).

5 Miaczynska, M. et al. APPL proteins link Rab5 to nuclear signal transduction via an endosomal compartment. Cell 116, 445-456, doi:10.1016/s0092-8674(04)00117-5 (2004).

6 Carpenter, G. \& Liao, H. J. Receptor tyrosine kinases in the nucleus. Cold Spring Harb Perspect Biol 5, a008979, doi:10.1101/cshperspect.a008979 (2013).

7 Chaumet, A. et al. Nuclear envelope-associated endosomes deliver surface proteins to the nucleus. Nat Commun 6, 8218, doi:10.1038/ncomms9218 (2015).

8 Shah, P., Chaumet, A., Royle, S. J. \& Bard, F. A. The NAE Pathway: Autobahn to the Nucleus for Cell Surface Receptors. Cells 8, doi:10.3390/cells8080915 (2019).

9 Lange, A. et al. Classical nuclear localization signals: definition, function, and interaction with importin alpha. J Biol Chem 282, 5101-5105, doi:10.1074/jbc.R600026200 (2007).

10 Kosugi, S., Hasebe, M., Tomita, M. \& Yanagawa, H. Systematic identification of cell cycledependent yeast nucleocytoplasmic shuttling proteins by prediction of composite motifs. Proc Natl Acad Sci U S A 106, 10171-10176, doi:10.1073/pnas.0900604106 (2009).

11 Gundry, C. et al. Phosphorylation of Rab-coupling protein by LMTK3 controls Rab14dependent EphA2 trafficking to promote cell:cell repulsion. Nat Commun 8, 14646, doi:10.1038/ncomms14646 (2017).

12 Esnault, C. et al. Rho-actin signaling to the MRTF coactivators dominates the immediate transcriptional response to serum in fibroblasts. Genes Dev 28, 943-958, doi:10.1101/gad.239327.114 (2014).

13 Gualdrini, F. et al. SRF Co-factors Control the Balance between Cell Proliferation and Contractility. Mol Cell 64, 1048-1061, doi:10.1016/j.molcel.2016.10.016 (2016).

14 Posern, G. \& Treisman, R. Actin' together: serum response factor, its cofactors and the link to signal transduction. Trends Cell Biol 16, 588-596, doi:10.1016/j.tcb.2006.09.008 (2006).

15 Hiramoto-Yamaki, N. et al. Ephexin4 and EphA2 mediate cell migration through a RhoGdependent mechanism. J Cell Biol 190, 461-477, doi:10.1083/jcb.201005141 (2010).

16 Mouilleron, S., Langer, C. A., Guettler, S., McDonald, N. Q. \& Treisman, R. Structure of a pentavalent G-actin*MRTF-A complex reveals how G-actin controls nucleocytoplasmic shuttling of a transcriptional coactivator. Sci Signal 4, ra40, doi:10.1126/scisignal.2001750 (2011).

17 Baarlink, C., Wang, H. \& Grosse, R. Nuclear actin network assembly by formins regulates the SRF coactivator MAL. Science 340, 864-867, doi:10.1126/science.1235038 (2013).

18 Posern, G., Sotiropoulos, A. \& Treisman, R. Mutant actins demonstrate a role for unpolymerized actin in control of transcription by serum response factor. Mol Biol Cell 13, 4167-4178, doi:10.1091/mbc.02-05-0068 (2002).

19 Stuven, T., Hartmann, E. \& Gorlich, D. Exportin 6: a novel nuclear export receptor that is specific for profilin.actin complexes. EMBO J 22, 5928-5940, doi:10.1093/emboj/cdg565 (2003).

20 Le, H. Q. et al. Mechanical regulation of transcription controls Polycomb-mediated gene silencing during lineage commitment. Nat Cell Biol 18, 864-875, doi:10.1038/ncb3387 (2016).

21 Van Troys, M. et al. Ins and outs of ADF/cofilin activity and regulation. Eur J Cell Bio/ 87, 649667, doi:10.1016/j.ejcb.2008.04.001 (2008). 
22 Dopie, J., Skarp, K. P., Rajakyla, E. K., Tanhuanpaa, K. \& Vartiainen, M. K. Active maintenance of nuclear actin by importin 9 supports transcription. Proc Natl Acad Sci U S A 109, E544-552, doi:10.1073/pnas.1118880109 (2012).

23 Hetrick, B., Han, M. S., Helgeson, L. A. \& Nolen, B. J. Small molecules CK-666 and CK-869 inhibit actin-related protein $2 / 3$ complex by blocking an activating conformational change. Chem Biol 20, 701-712, doi:10.1016/j.chembiol.2013.03.019 (2013).

24 Ross-Macdonald, P. et al. Identification of a nonkinase target mediating cytotoxicity of novel kinase inhibitors. Mol Cancer Ther 7, 3490-3498, doi:10.1158/1535-7163.MCT-08-0826 (2008).

25 Roberts, M., Barry, S., Woods, A., van der Sluijs, P. \& Norman, J. PDGF-regulated rab4dependent recycling of alphavbeta3 integrin from early endosomes is necessary for cell adhesion and spreading. Curr Biol 11, 1392-1402, doi:10.1016/s0960-9822(01)00442-0 (2001).

26 Caswell, P. T. et al. Rab-coupling protein coordinates recycling of alpha5beta1 integrin and EGFR1 to promote cell migration in 3D microenvironments. J Cell Biol 183, 143-155, doi:10.1083/jcb.200804140 (2008).

27 Cox, J. \& Mann, M. MaxQuant enables high peptide identification rates, individualized p.p.b.range mass accuracies and proteome-wide protein quantification. Nat Biotechnol 26, 13671372, doi:10.1038/nbt.1511 (2008).

28 Cox, J. et al. Andromeda: a peptide search engine integrated into the MaxQuant environment. J Proteome Res 10, 1794-1805, doi:10.1021/pr101065j (2011).

29 Resasco, D. C. et al. Virtual Cell: computational tools for modeling in cell biology. Wiley Interdiscip Rev Syst Biol Med 4, 129-140, doi:10.1002/wsbm.165 (2012). 


\section{Acknowledgements}

This work was funded by Cancer Research UK.

Many thanks to Drs. Christian Baarlink and Robert Grosse for the generous gift of H1299 cells expressing GFP-MRTF.

Many thanks to Prof. Anne Ridley for the generous gift of GFP-tagged RhoG expression vector.

\section{Author contributions}

Experimental work primary data analysis was performed by SM, MM, AP-G, LM, GB and JN. Computational modelling was designed and performed by MN. Bioinformatic analyses were performed by $\mathrm{HH}$ and $\mathrm{AH}$. $\mathrm{SZ}$ designed and supervised the proteomic approach to characterising the plasma membrane to nuclear traffickome. Experiments were conceived and designed by SM, MN, SZ and JN. SM and JN wrote the manuscript.

\section{Competing interests}

The authors declare no competing interests 


\section{Figure legends}

Figure 1: HGF induces nuclear-capture of EphA2 through its association with the nuclear import machinery.

a, $\mathrm{H} 1299$ cells were surface-labelled with NHS-SS-Biotin at $4^{\circ} \mathrm{C}$, and then warmed to $37^{\circ} \mathrm{C}$ in the presence (lower panel) or absence (centre panel) of HGF for 20 min or allowed to remain at $4^{\circ} \mathrm{C}$ (upper panel). Biotin remaining at the cell surface was removed by incubation with the membrane-impermeant reducing agent, MesNa at $4^{\circ} \mathrm{C}$, and distribution of biotinylated proteins visualised with fluorescent streptavidin (green) and counterstained with DAPI (blue) and Lamin

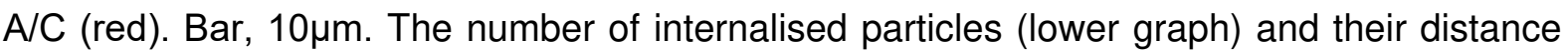
from the nuclear surface (upper graph) was quantified. The analysis was performed by obtaining 10 fields of view (FoV) for each condition in each independent experiment, in which we measured an average of 3 cells/FoV and analysed an average of 32 particles per cell. Values are mean \pm sem; ${ }^{* * *} \mathrm{P}<0.001$, one-way analysis of variance (ANOVA) of three independent experiments.

b, H1299 cells were SILAC-labelled with medium (M) or light (L) amino acids and surfacelabelled with NHS-SS-Biotin at $4^{\circ} \mathrm{C}$. Cells were warmed to $37^{\circ} \mathrm{C}$ in the presence of HGF (light labelled cells) or allowed to remain at $4^{\circ} \mathrm{C}$ (medium labelled cells). Following this, nuclei were purified, biotinylated proteins isolated from these using streptavidin beads and the proteome of isolates analysed by mass spectrometry (see schematic). The scatter plot indicates the resulting SILAC ratios of the biotinylated nuclear-associated proteome (+HGF / -HGF) from two independent experiments ((1) and (2)) plotted on the $\mathrm{y}$ and $\mathrm{x}$-axes respectively. Biotinylated proteins which are enriched in the nuclear fraction in response to HGF are represented in the upper right-hand quadrant. An aliquot of the cells which remained at $4^{\circ} \mathrm{C}$ were not fractionated and these were used to determine the surface proteome and proteins which are present in the surface proteome and which have, therefore, moved from the plasma membrane to the nuclear fraction are highlighted by red dots.

c, Surface-labelled $\mathrm{H} 1299$ cells were warmed to $37^{\circ} \mathrm{C}$ in the absence or presence of HGF for the indicated times to allow internalisation. Nuclei were purified and the presence of biotinylated EphA2 in these purified nuclei was determined using a capture-ELISA (left graph) and by immunoflourescence followed by super-resolution microscopy (right panels, Bar $5 \mu \mathrm{m}$ ). Data are expressed at the proportion of surface-labelled EphA2 which is translocated to the nuclear preparation. Values are mean \pm sem, ${ }^{* * * *} \mathrm{P}<0.0001$, repeated measures two-way analysis of variance (ANOVA) of three independent experiments. 
d, H1299 cells were transfected with siRNAs targeting EphA2 (siEphA2) or a non-targeting control (nt) and surface-labelled at $4^{\circ} \mathrm{C}$. Cells were then warmed to $37^{\circ} \mathrm{C}$ in the presence or absence of HGF and the number and distribution of internalised biotinylated particles determined with respect to the nuclear surface as for (a). Values are mean \pm sem, ${ }^{\star * *} \mathrm{P}<0.001$, one-way analysis of variance (ANOVA) of three independent experiments.

e, H1299 cells were transfected with siRNAs targeting Rab17 (siRab17) or non-targeting control (nt) and surface-labelled with NHS-SS-Biotin at $4^{\circ} \mathrm{C}$. Cell were warmed to $37^{\circ} \mathrm{C}$ for the indicated times in the absence or presence of HGF. Biotin remaining at the cell surface was removed by incubation with MesNa at $4^{\circ} \mathrm{C}$ and the quantity of biotinylated EphA2 determined using capture-ELISA. The proportion of internalized EphA2 is expressed as $\%$ of the pool of the receptor labelled at the cell surface. Values are mean \pm sem; ${ }^{*} p<0.05$ one-way analysis of variance (ANOVA) of four independent experiments.

f, Cells transfected with siRNAs targeting Rab17 (siRab17) or non-targeting control (nt) were incubated in the presence or absence of HGF for $5 \mathrm{~min}$. Nuclei were purified and the presence of EphA2 and Rab17 in these purified nuclei was determined using western blotting with histone $\mathrm{H} 2 \mathrm{a}$ as loading control.

g, Cells expressing EphA2-GFP and Rab17-mCherry were incubated with HGF for 5 min. Nuclei were purified and the distribution of GFP and mCherry visualised using super-resolution fluorescence microscopy (Bar $10 \mu \mathrm{m})$.

$\mathbf{h}$, Schematic diagram of the primary sequence of EphA2 with respect to its orientation in the endosomal membrane showing its predicted juxtamembrane NLS sequence and the amino acid substitutions deployed to generate the NLS1 and NLS2 mutants.

i, H1299 cells were transfected with EphA2-GFP, EphA2 NLS1-GFP or EphA2 NLS2-GFP and incubated in the presence or absence of HGF for $20 \mathrm{~min}$. Cells were lysed and EphA2GFPs immunoprecipitated using magnetic beads conjugated to an antibody recognising GFP. The presence of importins $-\alpha 5$ and $-\beta 1$ in the immunoprecipitates was determined using Western blotting. Blots are representative of 4 independent experiments.

j, H1299 cells were transfected with EphA2-GFP and the indicted NLS mutants of EphA2GFP. Cells were treated with HGF for the indicated times and delivery of the GFP-tagged EphA2s to the nuclear fraction was determined using capture-ELISA as for (c). Values are mean \pm sem, ${ }^{* \star * *} \mathrm{P}<0.0001$; ${ }^{* *} \mathrm{P}<0.001$; ${ }^{*} \mathrm{P}<0.05$ repeated measures two-way analysis of variance (ANOVA) of five independent experiments. 


\section{Figure 2: Nuclear-capture of EphA2 drives SRF/MRTF gene expression and cell scattering.}

a-c, Primary mouse cell lines were derived from pancreatic adenocarcinoma (PDAC) from KPC (Pdx1-Cre, Kras ${ }^{\mathrm{G} 12 \mathrm{D} /+}, \mathrm{p}^{2} 3^{\mathrm{R} 172 \mathrm{H} /+}$, EphA2 $\left.2^{-/}\right)$mice. EphA2 knockout cells stably expressing wild-type GFP-tagged EphA2 (EphA2 ${ }^{--}+$WT), or GFP-tagged EphA2s with mutated NLS sequences (EphA2 $2^{-/}+\mathrm{NLS} 1$ and $\left.E p h A 2^{-/-}+\mathrm{NLS} 2\right)$ or empty vector control $\left(E p h A 2^{-/}\right)$, and expression of EphA2s confirmed by Western blotting (a; right panel). PDAC cells were plated onto the underside of transwells containing Matrigel plugs and allowed to migrate into the plugs towards a gradient of HGF and serum for $72 \mathrm{hr}$, and then visualised by Calcein-AM followed by confocal microscopy. Optical sections were taken every $15 \mu \mathrm{m}$ and consecutive images are displayed as a series running from left to right (a; left panel). Cell invasion at the indicated distances into the plug was quantified and expressed as a $\%$ of the total quantity of fluorescent cells in the plug (a; centre panel). PDAC cells were plated onto glass surfaces and

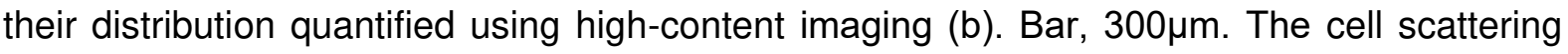
index (\% of objects not found in clusters of 5 or more cells) is displayed in the box and whisker plot (c). ${ }^{* \star *} \mathrm{P}<0.0001$, one-way ANOVA.

d, H1299 cells were transfected with siRNAs targeting EphA2 (siEphA2), Rab17 (siRab17) or non-targeting control (nt), in combination with wild-type (WT) or the indicted NLS mutants of EphA2-GFP as indicated. Cells were plated onto glass surfaces and allowed to form colonies of approx. 4 cells/colony and then challenged with HGF as indicated. Cell scattering was quantified using ImageJ and is expressed as the accumulated distance travelled over $8 \mathrm{hr}$. ${ }^{* * *} \mathrm{P}<0.001$, one-way analysis of variance (ANOVA) of 4 independent experiments.

e, H1299 cells were transfected with siRNAs targeting EphA2 (siEphA2) or non-targeting control (nt). Cells were then challenged with HGF for $2 \mathrm{hr}$, lysed and gene expression analysed by RNAseq. Each spot represents mRNAs which are either significantly up- or down-regulated by HGF. Thus, mRNAs whose HGF-dependent expression is not influenced by EphA2 knockdown lie along the red diagonal line. There are 25 mRNAs whose HGF-dependent regulation is opposed by EphA2 knockdown (following implementation of a cut-off excluding fold changes of $<\log 2$ of 1 ). These are denoted by red dots which lie above the red diagonal line, and their identities are indicated. The heatmap displays the HGF-dependent regulation of these 25 EphA2-dependent mRNAs (SRF target genes are denoted in red text). 3 independent experiments.

f, $\mathrm{H} 1299$ cells were transfected with an siRNA targeting EphA2 in combination with wild-type EphA2-GFP (WT) or NLS mutants of EphA2-GFP (NLS1 and NLS2). Cells were challenged with HGF or were left unchallenged for 20 min and the levels of the indicated mRNAs (FosB; 
JunB; ATF3; and Zfp36) were determined using qPCR. The HGF-induced fold change in mRNA level is plotted on the $y$-axis, each bar is the mean of at least 4 independent experiments \pm sem, ${ }^{*} P<0.05$ paired t-test. Paired data are denoted by dots of the same colour.

g, H1299 cells were transfected with an siRNA targeting EphA2 (siEphA2) or a non-targeting control (nt) and then incubated in the presence or absence of HGF for 20 min. Cells were then lysed and DNA sheared by sonication. MRTF (upper panels) or SRF (lower panels) were immunoprecipitated from lysates and the and binding of these to the promoter of the indicated genes was determined using qPCR. The HGF-induced fold change in immunoprecipitated genes is plotted on the $y$-axis. Values are mean \pm sem, ${ }^{* *} \mathrm{P}<0.0001,{ }^{*} \mathrm{P}<0.005$ repeated measures one way ANOVA.

Figure 3: Nuclear-capture of EphA2 is required for perinuclear actin polymerisation and depletion of nuclear G-actin.

a, H1299 cells were transfected with wild-type EphA2-GFP (WT) or an NLS mutant of EphA2GFP (NLS2) (green) in combination with mCherry-Lifeact (red) in the presence or absence of an siRNA targeting RhoG (siRhoG). Cells were challenged with HGF and monitored using high resolution time-lapse fluorescence imaging and movies collected (Supplementary movies 4 -6). Bar, $10 \mu \mathrm{m}$. Stills (captured at the indicated times following HGF addition) from these movies are presented in the inserts and the mCherry-Lifeact channel is displayed in these. The intensity of mCherry-Lifeact fluorescence in the perinuclear region was quantified and is expressed as the fluorescence change evoked by HGF addition as a function of the average fluorescence prior to HGF addition. The solid lines represent the mean fluorescence change in $n>7$ cells each from an independent biological repeat, and the dotted lines represent the sem. Coloured asterisks represent comparisons of the WT+HGF (blue) with WT-HGF (grey), siRhoG+HGF (red) or NLS2+HGF (green) P<0.05, 2-way ANOVA.

b, $\mathrm{H} 1299$ cells were incubated in the presence or absence of HGF for 20 min and then fixed. Fixed cells were stained with fluorescence DNase I to visualise G-actin (G-actin; red) and counterstained with phalloidin (green) and DAPI (blue). The periphery of the nucleus is denoted in red.

c, H1299 cells were transfected with siRNAs targeting EphA2 (siEphA2) or non-targeting control (nt) in combination with either wild-type EphA2-GFP (WT) or EphA2 NLS2-GFP (NLS2). Cells were then challenged with HGF for 20 min (+HGF) or left unchallenged (-HGF) and nuclei purified from these. Purified nuclei were fixed and stained with fluorescent DNasel to visualise G-actin and analysed using flow cytometry. Fluorescence histograms are 
displayed in the upper panels. In the box and whisker plots the geometrical mean fluorescence was calculated, and data are shown as the ratio of the geometrical mean value of HGF-treated cells as a function of unchallenged cells (+HGF/-HGF). ${ }^{*} \mathrm{P}<0.05$, unpaired t-test analysis of three (left panel) and five (right panel) independent experiments.

d, H1299 cells stably expressing MRTF-GFP were transfected with either siRNAs targeting EphA2 (siEphA2), RhoG (siRhoG) or non-targeting control (nt), or non-polymerisable actin (R62D actin) or nuclear-targeted non-polymerisable actin (NLS R62D actin) mutants or vector control. Cells were incubated in the absence (-HGF) or presence (+HGF) of HGF for 20 min, stained with DAPI to visualise nuclei and imaged by high-content automated microscopy. The distribution of MRTF-GFP was determined using high-content analysis and expressed as the ratio of MRTF-GFP in the nucleus as a function of that present in the cytoplasm. ${ }^{* * * *} \mathrm{P}<0.0001$, one-way ANOVA.

e, $\mathrm{H} 1299$ cells were transfected with non-polymerisable actin (R62D actin) or vector control. Cells were plated onto glass surfaces and cell scattering in the presence and absence of HGF determined as for Fig. $2 c$. ${ }^{* * *} \mathrm{P}<0.001$, one-way analysis of variance (ANOVA) of 4 independent experiments.

\section{Figure 4: The role of cofilin phosphorylation in controlling nucleocytoplasmic actin dynamics and gene expression}

a, Schematic summary of pathways downstream of EphA2 likely to influence nucleocytoplasmic actin dynamics. Cytoplasmic $\mathrm{G}$-actin $\left(\mathrm{G}_{\mathrm{c}}\right)$ may be polymerised into $\mathrm{F}$-actin $\left(F_{c}\right)$ in the juxtanuclear region. $G$-actin associates with cofilin in the cytoplasm and the cofilinactin complex may enter the nucleus. However, phosphorylation of cofilin at $\mathrm{Ser}^{3}$ opposes its association with G-actin. Nuclear G-actin associates with profilin and the resulting profilin-actin complex leaves the nucleus via exportin-6. The rates of cytoplasmic actin polymerisation and cofilin phosphorylation are denoted by $E_{c} \mu 1$ and $E_{c} \mu 2$ respectively.

b, H1299 cells were transfected with siRNAs targeting EphA2 (siEphA2), RhoG (siRhoG) or non-targeting siRNA (nt) in combination with expression vectors for wild-type EphA2 (WT), EphA2 NLS1 (NLS1) or EphA2 NLS2 (NLS2). Primary mouse cell lines were derived from pancreatic adenocarcinoma (PDAC) from KPC mice (KPC EphA2 ${ }^{+/+}$) or EphA2 knockout KPC mice (KPC EphA2 $2^{-/}$). Cells were incubated in the absence or presence of HGF for 20 min and levels of cofilin and phosphocofilin (cofilin pSer3) determined by Western blotting with actin as loading control. 
c, A computational approach was used to model nucleocytoplasmic actin dynamics according to the schematic in Fig. 4a. The images depict model cells in which the nuclear envelope is clearly shown and the alterations to G-actin levels are represented by the Viridis colour palette. The images represent three time points from the movie presented in supplementary movie 7 . The influence of increasing the rates of actin polymerisation $\left(E_{c} \mu 1\right)$ and cofilin phosphorylation $\left(E_{c} \mu 2\right)$ on depletion of nuclear G-actin was evaluated. The left-hand images and graph (black line) depict predicted alterations to G-actin distribution following activation of both juxtanuclear actin polymerisation and cofilin phosphorylation effected by increasing the rates $E_{c} \mu 1$ and $E_{c} \mu 2$ to values greater than zero $\left(E_{c} \mu 1>0\right.$ and $\left.E_{c} \mu 2>0\right)$. The centre image panels and graph (red line) depict alterations to G-actin distribution occurring following activation of actin polymerisation $\left(E_{c} \mu 1>0\right)$, but not cofilin phosphorylation $\left(E_{c} \mu 2=0\right)$. The right-hand image panels and blue line in the graph depict predicted G-actin dynamics following activation of cofilin phosphorylation $\left(E_{c} \mu 2>0\right)$, but not actin polymerisation $\left(E_{c} \mu 1=0\right)$.

d, $\mathrm{H} 1299$ cells were challenged with HGF or left unchallenged for $20 \mathrm{~min}$ in the absence or presence of LIMK inhibitor (BMS5), and levels of the indicated mRNAs (FosB; JunB; ATF3; and Zfp36) determined using qPCR. The HGF-induced fold change in mRNA level is plotted on the $y$-axis, each bar is the mean of at least 5 independent experiments \pm sem, ${ }^{* *} P<0.001$ paired t-test. Paired data are denoted by dots of the same colour.

e, Cells were plated onto glass surfaces and cell scattering in the presence and absence of HGF and LIMK inhibitor (BMS5) determined as for Fig. 2c. ${ }^{* *} \mathrm{P}<0.001$, one-way analysis of variance (ANOVA) of 4 independent experiments.

f, cMET drives Rab17-dependent endocytosis of EphA2 [1]. EphA2 endosomes are then transported centripetally under control of Rab17 [2] and become physically attached to, or 'captured' by, the nucleus by an interaction formed between the nuclear import machinery and a nuclear localisation sequence located in EphA2's cytodomain [3]. This nuclear-capture event, in turn, drives actin polymerisation which is restricted to the juxtanuclear region [4] and LIMK-driven phosphorylation of cofilin [5], and both events are dependent on the RhoG GTPase. Phosphorylation of cofilin opposes nuclear import of cofilin-actin, leading to depletion of G-actin from the nucleus [6] which, in turn, activates transcription of MRTF/SRF-target genes [7] to implement cell scattering and invasion. 
Marco et al., Figure 1

a

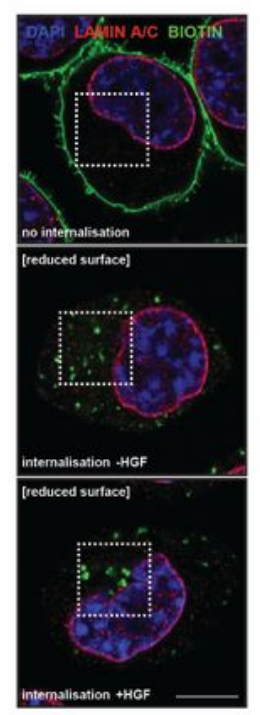

C

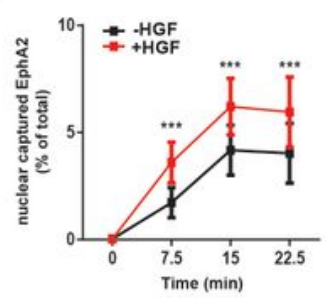

e

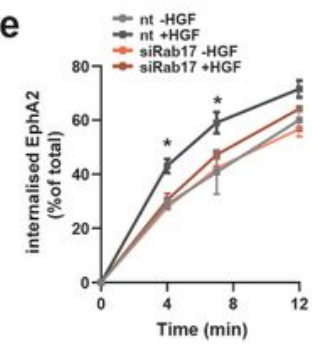

h

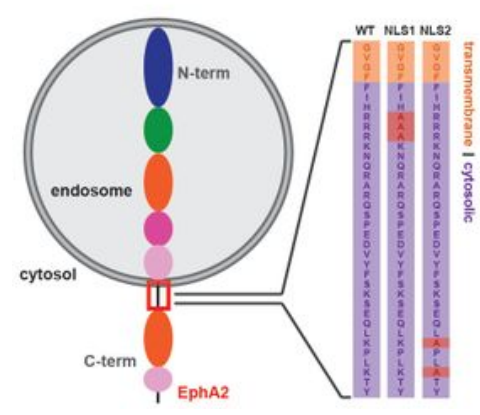

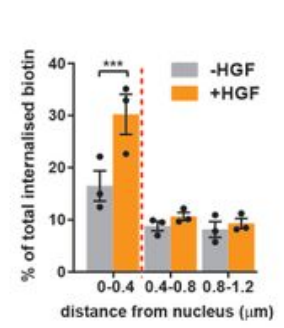

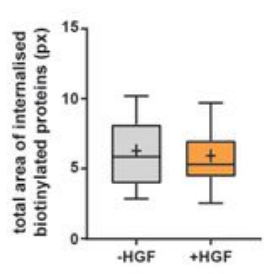

f

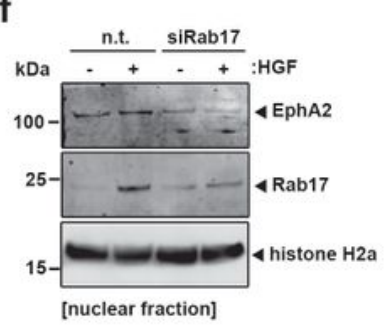

b

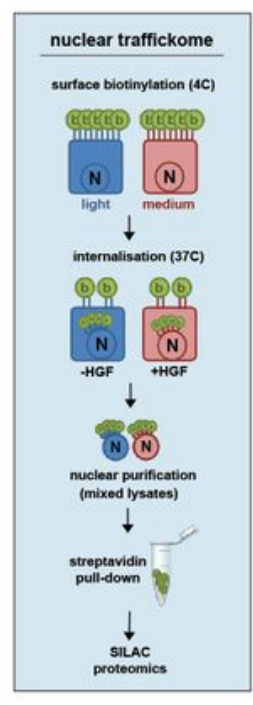

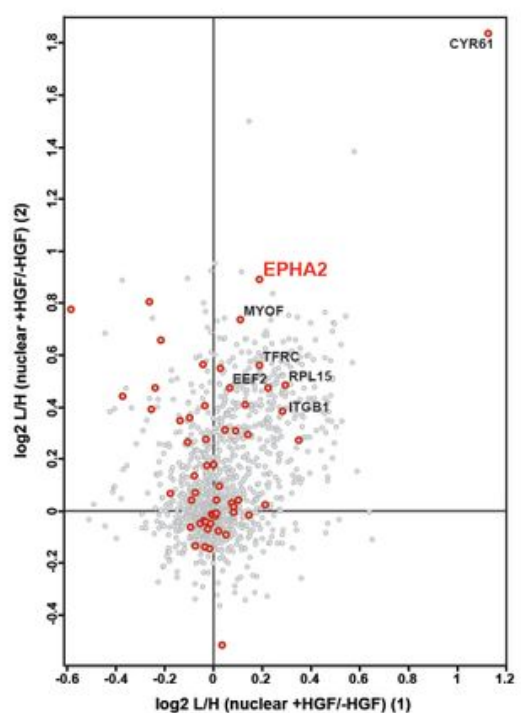

d

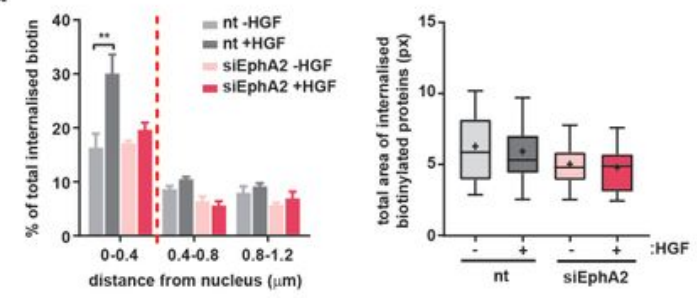

g

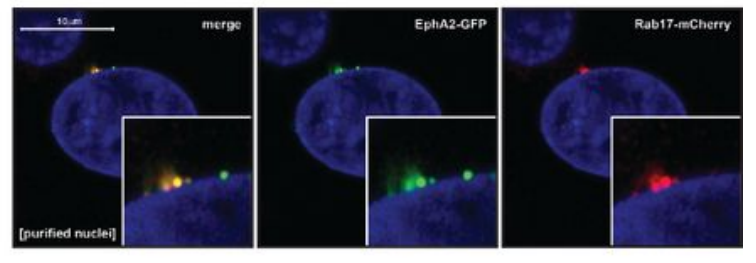

i
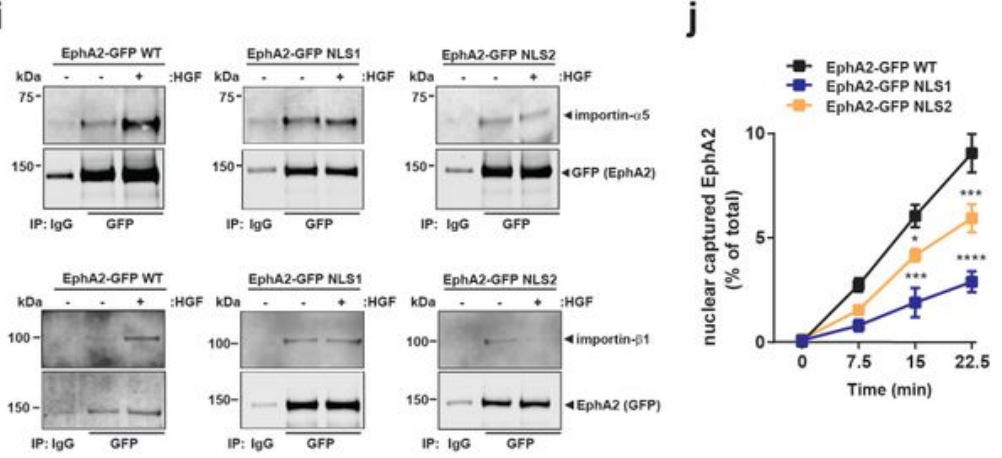

Figure 1

HGF induces nuclear-capture of EphA2 through its association with the nuclear import machinery. a, $\mathrm{H} 1299$ cells were surface-labelled with NHS-SS-Biotin at $4^{\circ} \mathrm{C}$, and then warmed to $37^{\circ} \mathrm{C}$ in the presence (lower panel) or absence (centre panel) of HGF for 20 min or allowed to remain at $4^{\circ} \mathrm{C}$ (upper panel). 
Biotin remaining at the cell surface was removed by incubation with the membrane-impermeant reducing agent, MesNa at $4^{\circ} \mathrm{C}$, and distribution of biotinylated proteins visualised with fluorescent streptavidin (green) and counterstained with DAPI (blue) and Lamin A/C (red). Bar, 10 $\mu \mathrm{m}$. The number of internalised particles (lower graph) and their distance from the nuclear surface (upper graph) was quantified. The analysis was performed by obtaining 10 fields of view (FoV) for each condition in each independent experiment, in which we measured an average of 3 cells/FoV and analysed an average of 32 particles per cell. Values are mean $\pm s e m ; * * * P<0.001$, one-way analysis of variance (ANOVA) of three independent experiments. b, H1299 cells were SILAC-labelled with medium (M) or light (L) amino acids and surfacelabelled with NHS-SS-Biotin at $4^{\circ} \mathrm{C}$. Cells were warmed to $37^{\circ} \mathrm{C}$ in the presence of HGF (light labelled cells) or allowed to remain at $4^{\circ} \mathrm{C}$ (medium labelled cells). Following this, nuclei were purified, biotinylated proteins isolated from these using streptavidin beads and the proteome of isolates analysed by mass spectrometry (see schematic). The scatter plot indicates the resulting SILAC ratios of the biotinylated nuclear-associated proteome (+HGF / -HGF) from two independent experiments ((1) and (2)) plotted on the $y$ and $x$-axes respectively. Biotinylated proteins which are enriched in the nuclear fraction in response to HGF are represented in the upper right-hand quadrant. An aliquot of the cells which remained at $4^{\circ} \mathrm{C}$ were not fractionated and these were used to determine the surface proteome and proteins which are present in the surface proteome and which have, therefore, moved from the plasma membrane to the nuclear fraction are highlighted by red dots. c, Surface-labelled $\mathrm{H} 1299$ cells were warmed to $37^{\circ} \mathrm{C}$ in the absence or presence of HGF for the indicated times to allow internalisation. Nuclei were purified and the presence of biotinylated EphA2 in these purified nuclei was determined using a capture-ELISA (left graph) and by immunoflourescence followed by super-resolution microscopy (right panels, Bar $5 \mu \mathrm{m}$ ). Data are expressed at the proportion of surface-labelled EphA2 which is translocated to the nuclear preparation. Values are mean \pm sem, $* \star \star * P<0.0001$, repeated measures two-way analysis of variance (ANOVA) of three independent experiments. d, H1299 cells were transfected with siRNAs targeting EphA2 (siEphA2) or a non-targeting control (nt) and surface-labelled at $4^{\circ} \mathrm{C}$. Cells were then warmed to $37^{\circ} \mathrm{C}$ in the presence or absence of HGF and the number and distribution of internalised biotinylated particles determined with respect to the nuclear surface as for (a). Values are mean \pm sem, ${ }^{\star \star *} \mathrm{P}<0.001$, one-way analysis of variance (ANOVA) of three independent experiments. e, H1299 cells were transfected with siRNAs targeting Rab17 (siRab17) or non-targeting control (nt) and surface-labelled with NHS-SS-Biotin at $4^{\circ} \mathrm{C}$. Cell were warmed to $37^{\circ} \mathrm{C}$ for the indicated times in the absence or presence of HGF. Biotin remaining at the cell surface was removed by incubation with MesNa at $4^{\circ} \mathrm{C}$ and the quantity of biotinylated EphA2 determined using capture-ELISA. The proportion of internalized EphA2 is expressed as \% of the pool of the receptor labelled at the cell surface. Values are mean \pm sem; ${ }^{*} p<0.05$ one-way analysis of variance (ANOVA) of four independent experiments. $f$, Cells transfected with siRNAs targeting Rab17 (siRab17) or non-targeting control (nt) were incubated in the presence or absence of HGF for 5 min. Nuclei were purified and the presence of EphA2 and Rab17 in these purified nuclei was determined using western blotting with histone $\mathrm{H} 2 \mathrm{a}$ as loading control. g, Cells expressing EphA2-GFP and Rab17-mCherry were incubated with HGF for 5 min. Nuclei were purified and the distribution of GFP and mCherry visualised using super-resolution fluorescence microscopy (Bar $10 \mu \mathrm{m})$. h, Schematic diagram of the primary sequence of EphA2 with respect to its orientation in the endosomal membrane showing its predicted 
juxtamembrane NLS sequence and the amino acid substitutions deployed to generate the NLS1 and NLS2 mutants. i, H1299 cells were transfected with EphA2-GFP, EphA2 NLS1-GFP or EphA2 NLS2-GFP and incubated in the presence or absence of HGF for $20 \mathrm{~min}$. Cells were lysed and EphA2-GFPs immunoprecipitated using magnetic beads conjugated to an antibody recognising GFP. The presence of importins - $\alpha 5$ and - $\beta 1$ in the immunoprecipitates was determined using Western blotting. Blots are representative of 4 independent experiments. j, H1299 cells were transfected with EphA2-GFP and the indicted NLS mutants of EphA2-GFP. Cells were treated with HGF for the indicated times and delivery of the GFP-tagged EphA2s to the nuclear fraction was determined using capture-ELISA as for (c). Values are mean \pm sem, ${ }^{\star \star \star \star} P<0.0001 ; * \star \star ~ P<0.001 ; * P<0.05$ repeated measures two-way analysis of variance (ANOVA) of five independent experiments. 
a
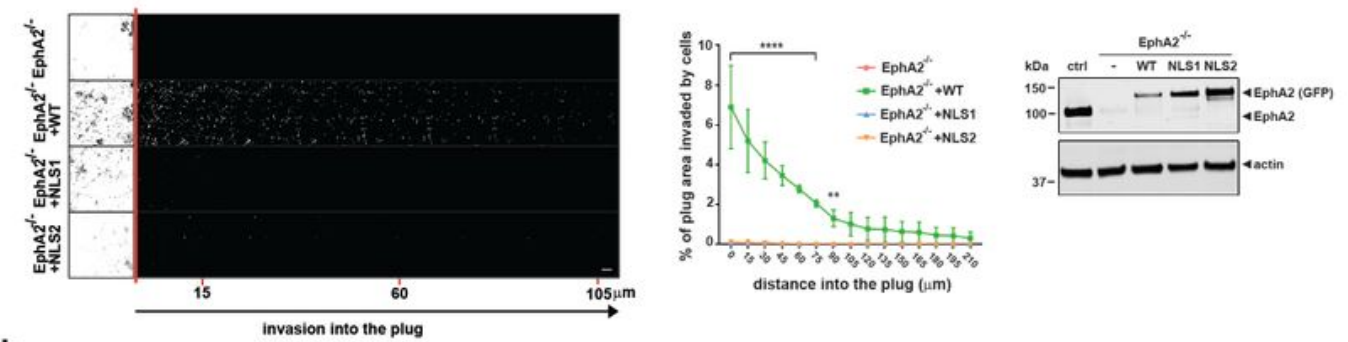

b
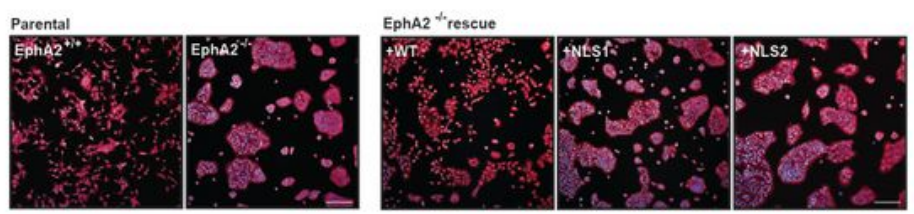

C

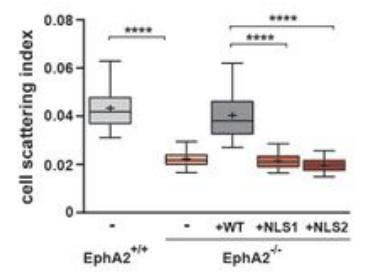

d

e
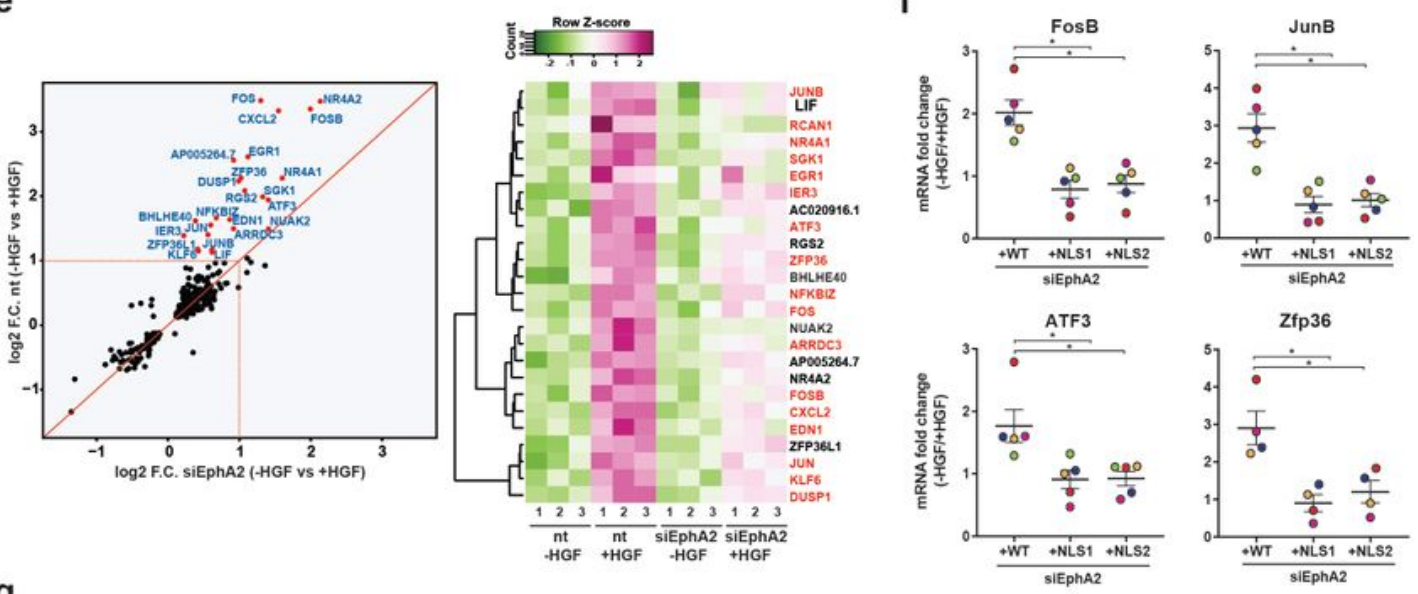

g

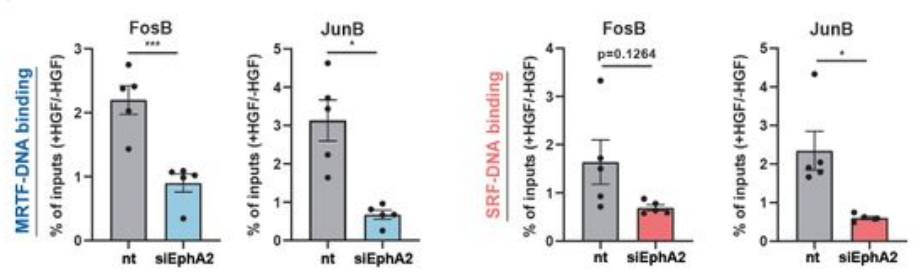

Figure 2

Nuclear-capture of EphA2 drives SRF/MRTF gene expression and cell scattering. a-c, Primary mouse cell lines were derived from pancreatic adenocarcinoma (PDAC) from KPC (Pdx1-Cre, KrasG12D/+, p53R172H/+, EphA2-/-) mice. EphA2 knockout cells stably expressing wild-type GFP-tagged EphA2 (EphA2-/- + WT), or GFP-tagged EphA2s with mutated NLS sequences (EphA2-/- + NLS1 and EphA2-/- + NLS2) or empty vector control (EphA2-/-), and expression of EphA2s confirmed by Western blotting (a; 
right panel). PDAC cells were plated onto the underside of transwells containing Matrigel plugs and allowed to migrate into the plugs towards a gradient of HGF and serum for $72 \mathrm{hr}$, and then visualised by Calcein-AM followed by confocal microscopy. Optical sections were taken every $15 \mu \mathrm{m}$ and consecutive images are displayed as a series running from left to right (a; left panel). Cell invasion at the indicated distances into the plug was quantified and expressed as a \% of the total quantity of fluorescent cells in the plug ( $a$; centre panel). PDAC cells were plated onto glass surfaces and their distribution quantified

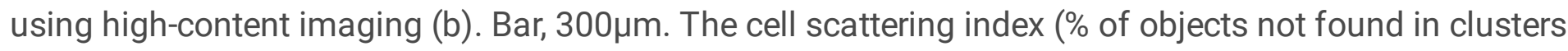
of 5 or more cells) is displayed in the box and whisker plot (c). ${ }^{\star} \star \star \star P<0.0001$, one-way ANOVA. d, H1299 cells were transfected with siRNAs targeting EphA2 (siEphA2), Rab17 (siRab17) or non-targeting control (nt), in combination with wild-type (WT) or the indicted NLS mutants of EphA2-GFP as indicated. Cells were plated onto glass surfaces and allowed to form colonies of approx. 4 cells/colony and then challenged with HGF as indicated. Cell scattering was quantified using Image $J$ and is expressed as the accumulated distance travelled over $8 \mathrm{hr}$. $* \star * P<0.001$, one-way analysis of variance (ANOVA) of 4 independent experiments. e, H1299 cells were transfected with siRNAs targeting EphA2 (siEphA2) or nontargeting control (nt). Cells were then challenged with HGF for $2 \mathrm{hr}$, lysed and gene expression analysed by RNAseq. Each spot represents mRNAs which are either significantly up- or down-regulated by HGF. Thus, mRNAs whose HGF-dependent expression is not influenced by EphA2 knockdown lie along the red diagonal line. There are 25 mRNAs whose HGF-dependent regulation is opposed by EphA2 knockdown (following implementation of a cut-off excluding fold changes of $<\log 2$ of 1 ). These are denoted by red dots which lie above the red diagonal line, and their identities are indicated. The heatmap displays the HGF-dependent regulation of these 25 EphA2-dependent mRNAs (SRF target genes are denoted in red text). 3 independent experiments. f, H1299 cells were transfected with an siRNA targeting EphA2 in combination with wild-type EphA2-GFP (WT) or NLS mutants of EphA2-GFP (NLS1 and NLS2). Cells were challenged with HGF or were left unchallenged for $20 \mathrm{~min}$ and the levels of the indicated mRNAs (FosB; JunB; ATF3; and Zfp36) were determined using qPCR. The HGF-induced fold change in mRNA level is plotted on the $y$-axis, each bar is the mean of at least 4 independent experiments \pm sem, ${ }^{*}<<0.05$ paired $t$ test. Paired data are denoted by dots of the same colour. g, H1299 cells were transfected with an siRNA targeting EphA2 (siEphA2) or a non-targeting control (nt) and then incubated in the presence or absence of HGF for 20 min. Cells were then lysed and DNA sheared by sonication. MRTF (upper panels) or SRF (lower panels) were immunoprecipitated from lysates and the and binding of these to the promoter of the indicated genes was determined using qPCR. The HGF-induced fold change in immunoprecipitated genes is plotted on the $y$-axis. Values are mean \pm sem, ${ }^{\star \star *} P<0.0001, * P<0.005$ repeated measures one way ANOVA. 
a

Marco et al., Figure 3
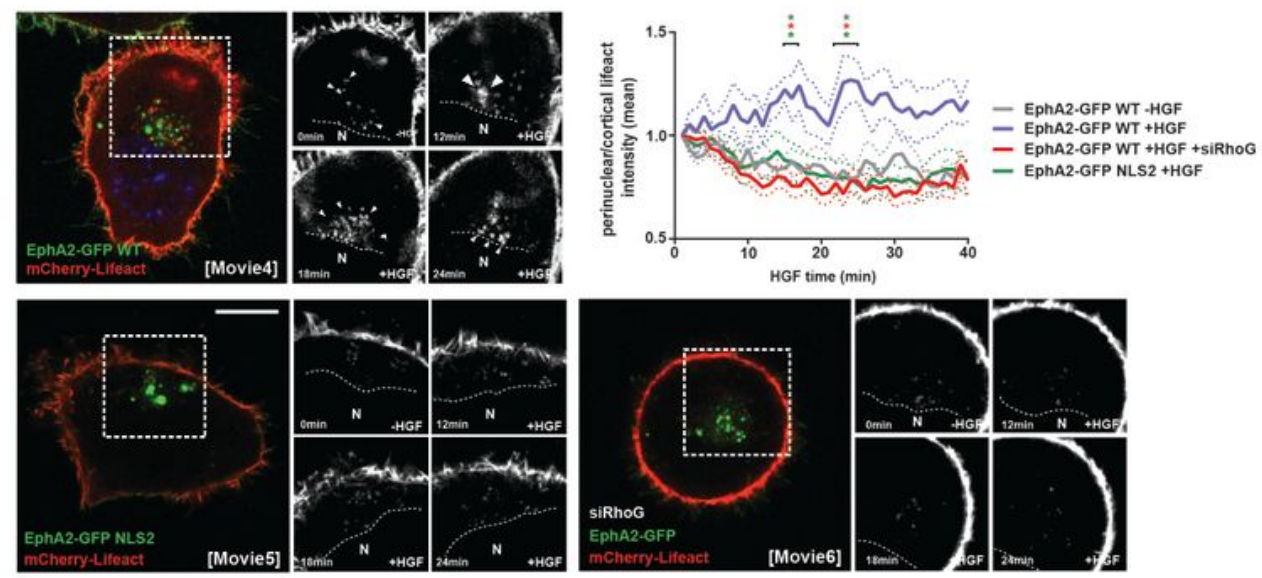

b

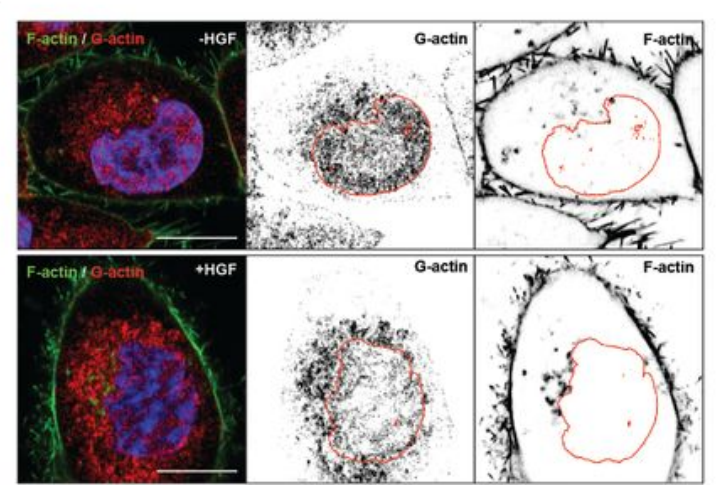

C
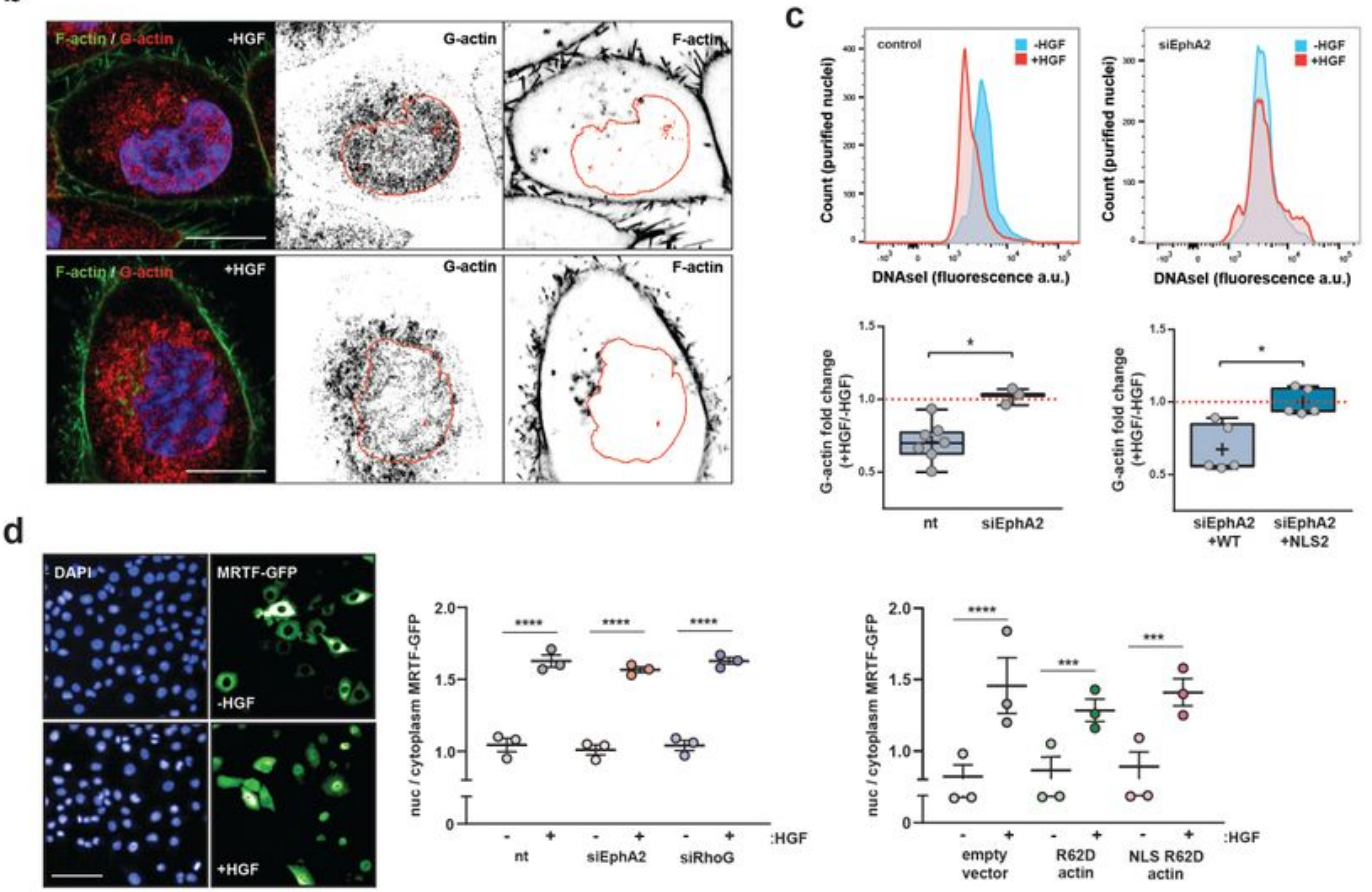

e
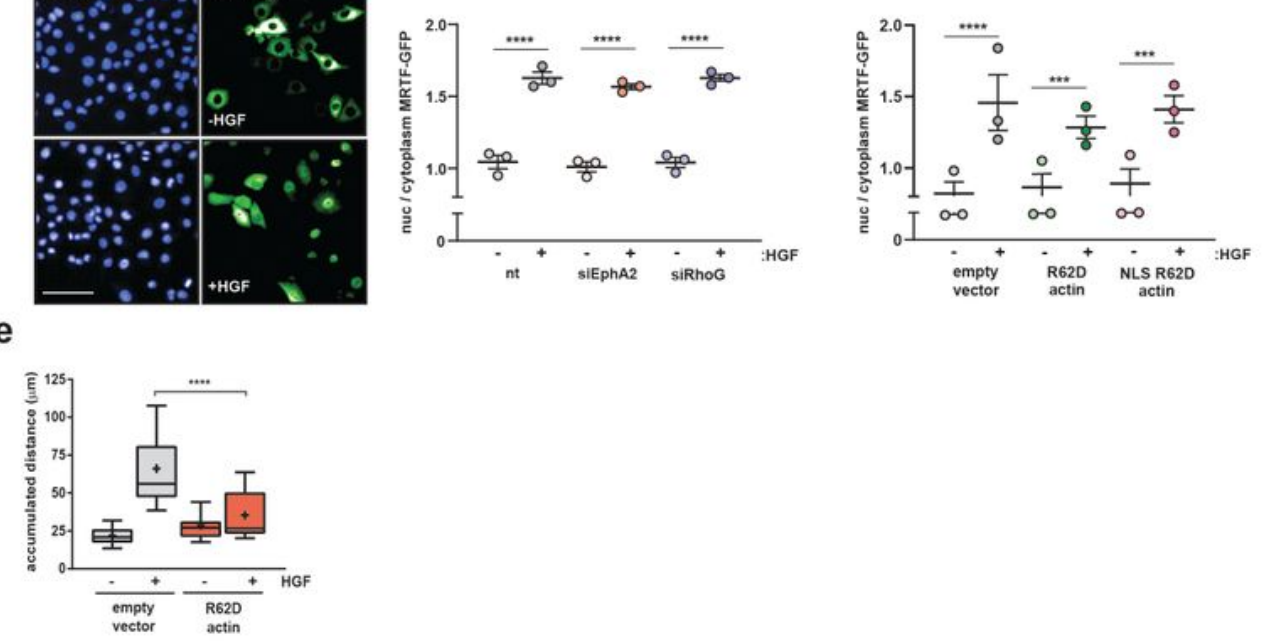

\section{Figure 3}

Figure 3: Nuclear-capture of EphA2 is required for perinuclear actin polymerisation and depletion of nuclear G-actin. a, H1299 cells were transfected with wild-type EphA2-GFP (WT) or an NLS mutant of EphA2-GFP (NLS2) (green) in combination with mCherry-Lifeact (red) in the presence or absence of an siRNA targeting RhoG (siRhoG). Cells were challenged with HGF and monitored using high resolution time-lapse fluorescence imaging and movies collected (Supplementary movies 4 -6). Bar, $10 \mu \mathrm{m}$. Stills 
(captured at the indicated times following HGF addition) from these movies are presented in the inserts and the mCherry-Lifeact channel is displayed in these. The intensity of mCherry-Lifeact fluorescence in the perinuclear region was quantified and is expressed as the fluorescence change evoked by HGF addition as a function of the average fluorescence prior to HGF addition. The solid lines represent the mean fluorescence change in $n>7$ cells each from an independent biological repeat, and the dotted lines represent the sem. Coloured asterisks represent comparisons of the WT+HGF (blue) with WT-HGF (grey), siRhoG+HGF (red) or NLS2+HGF (green) P<0.05, 2-way ANOVA. b, H1299 cells were incubated in the presence or absence of HGF for 20 min and then fixed. Fixed cells were stained with fluorescence DNase I to visualise G-actin (G-actin; red) and counterstained with phalloidin (green) and DAPI (blue). The periphery of the nucleus is denoted in red. c, $\mathrm{H} 1299$ cells were transfected with siRNAs targeting EphA2 (siEphA2) or non-targeting control (nt) in combination with either wild-type EphA2-GFP (WT) or EphA2 NLS2-GFP (NLS2). Cells were then challenged with HGF for 20 min (+HGF) or left unchallenged (-HGF) and nuclei purified from these. Purified nuclei were fixed and stained with fluorescent DNasel to visualise G-actin and analysed using flow cytometry. Fluorescence histograms are displayed in the upper panels. In the box and whisker plots the geometrical mean fluorescence was calculated, and data are shown as the ratio of the geometrical mean value of HGF-treated cells as a function of unchallenged cells (+HGF/HGF). ${ }^{*}<<0.05$, unpaired $t$-test analysis of three (left panel) and five (right panel) independent experiments. d, H1299 cells stably expressing MRTF-GFP were transfected with either siRNAs targeting EphA2 (siEphA2), RhoG (siRhoG) or non-targeting control (nt), or non-polymerisable actin (R62D actin) or nuclear-targeted non-polymerisable actin (NLS R62D actin) mutants or vector control. Cells were incubated in the absence (-HGF) or presence (+HGF) of HGF for 20 min, stained with DAPI to visualise nuclei and imaged by high-content automated microscopy. The distribution of MRTF-GFP was determined using high-content analysis and expressed as the ratio of MRTF-GFP in the nucleus as a function of that present in the cytoplasm. ${ }^{* \star \star *} \mathrm{P}<0.0001$, one-way ANOVA. e, H1299 cells were transfected with non-polymerisable actin (R62D actin) or vector control. Cells were plated onto glass surfaces and cell scattering in the presence and absence of HGF determined as for Fig. $2 c$. ${ }^{*} * \mathrm{P}<0.001$, one-way analysis of variance (ANOVA) of 4 independent experiments. 
a

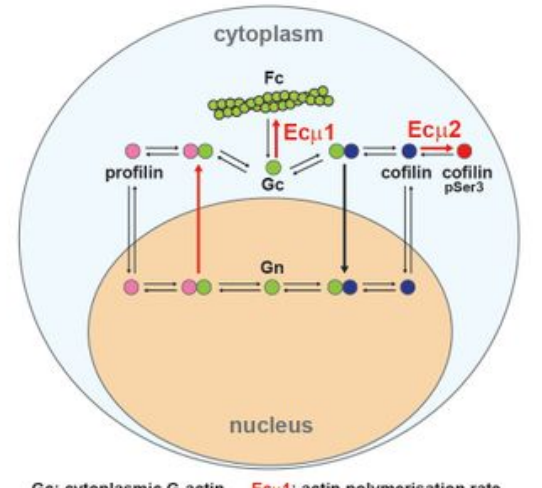

Gc: cytoplasmic G-actin Ecu1: actin polymerisation rate Gn: nuclear G-actin

Fc: cytoplasmic F-actin

C

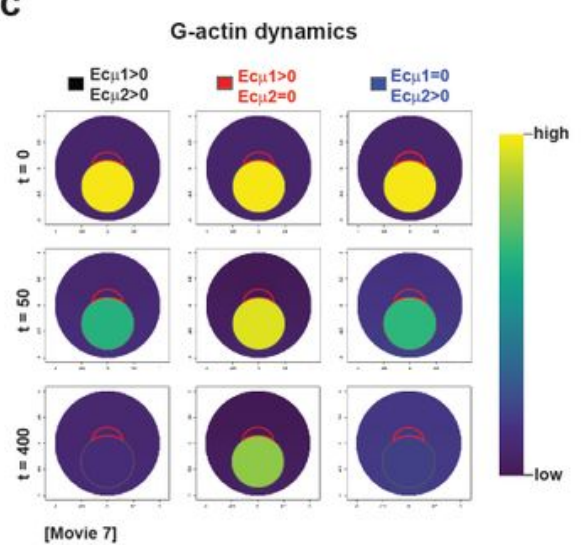

b
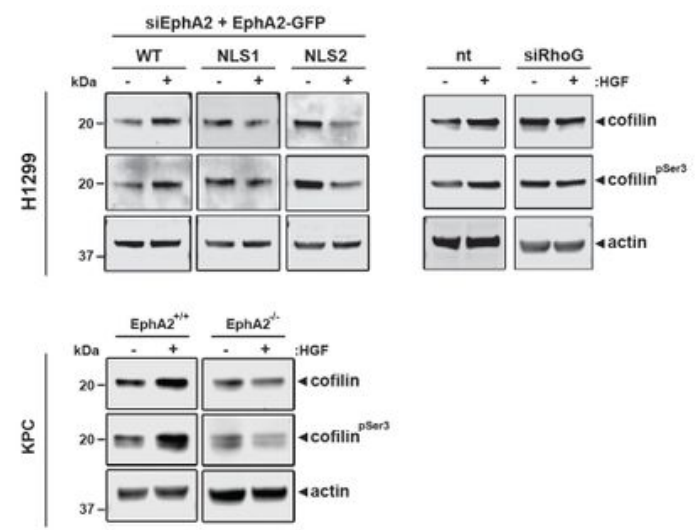

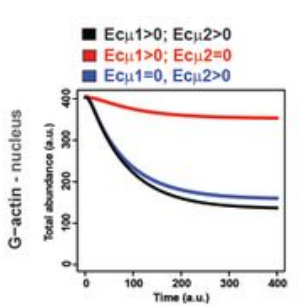

d
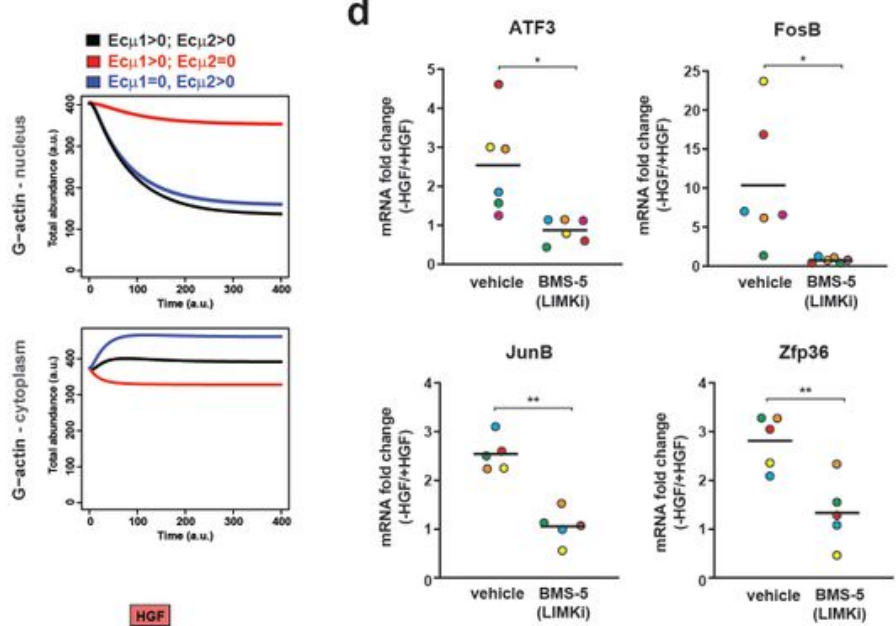

e
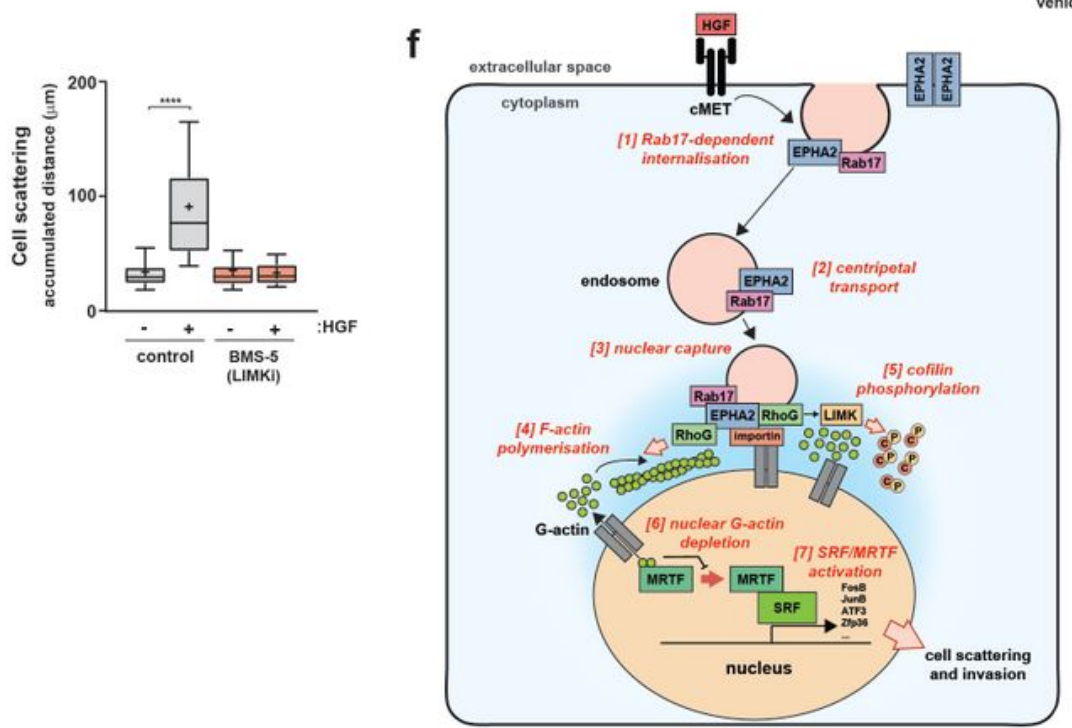

\section{Figure 4}

The role of cofilin phosphorylation in controlling nucleocytoplasmic actin dynamics and gene expression a, Schematic summary of pathways downstream of EphA2 likely to influence nucleocytoplasmic actin dynamics. Cytoplasmic G-actin (Gc) may be polymerised into F-actin (Fc) in the juxtanuclear region. Gactin associates with cofilin in the cytoplasm and the cofilin-actin complex may enter the nucleus. However, phosphorylation of cofilin at Ser3 opposes its association with G-actin. Nuclear G-actin 
associates with profilin and the resulting profilin-actin complex leaves the nucleus via exportin- 6 . The rates of cytoplasmic actin polymerisation and cofilin phosphorylation are denoted by Ec 1 and Ec 2 respectively. b, H1299 cells were transfected with siRNAs targeting EphA2 (siEphA2), RhoG (siRhoG) or non-targeting siRNA (nt) in combination with expression vectors for wild-type EphA2 (WT), EphA2 NLS1 (NLS1) or EphA2 NLS2 (NLS2). Primary mouse cell lines were derived from pancreatic adenocarcinoma (PDAC) from KPC mice (KPC EphA2+/+) or EphA2 knockout KPC mice (KPC EphA2-/-). Cells were incubated in the absence or presence of HGF for 20 min and levels of cofilin and phosphocofilin (cofilin pSer3) determined by Western blotting with actin as loading control. c, A computational approach was used to model nucleocytoplasmic actin dynamics according to the schematic in Fig. 4a. The images depict model cells in which the nuclear envelope is clearly shown and the alterations to G-actin levels are represented by the Viridis colour palette. The images represent three time points from the movie presented in supplementary movie 7 . The influence of increasing the rates of actin polymerisation (Ec 1 1) and cofilin phosphorylation (Ech2) on depletion of nuclear G-actin was evaluated. The left-hand images and graph (black line) depict predicted alterations to G-actin distribution following activation of both juxtanuclear actin polymerisation and cofilin phosphorylation effected by increasing the rates Ec 1 and $E c \mu 2$ to values greater than zero $(E c \mu 1>0$ and $E c \mu 2>0)$. The centre image panels and graph (red line) depict alterations to $\mathrm{G}$-actin distribution occurring following activation of actin polymerisation $(\mathrm{Ec} \mu 1>0)$, but not cofilin phosphorylation $(E c \mu 2=0)$. The right-hand image panels and blue line in the graph depict predicted G-actin dynamics following activation of cofilin phosphorylation $(E c \mu 2>0)$, but not actin polymerisation $(E c \mu 1=0)$. d, H1299 cells were challenged with HGF or left unchallenged for 20 min in the absence or presence of LIMK inhibitor (BMS5), and levels of the indicated mRNAs (FosB; JunB; ATF3; and Zfp36) determined using qPCR. The HGF-induced fold change in mRNA level is plotted on the y-axis, each bar is the mean of at least 5 independent experiments $\pm s e m, * * P<0.001$ paired $t$-test. Paired data are denoted by dots of the same colour. e, Cells were plated onto glass surfaces and cell scattering in the presence and absence of HGF and LIMK inhibitor (BMS5) determined as for Fig. $2 c$. ${ }^{* \star *} P<0.001$, one-way analysis of variance (ANOVA) of 4 independent experiments. $\mathrm{f}$, cMET drives Rab17-dependent endocytosis of EphA2 [1]. EphA2 endosomes are then transported centripetally under control of Rab17 [2] and become physically attached to, or 'captured' by, the nucleus by an interaction formed between the nuclear import machinery and a nuclear localisation sequence located in EphA2's cytodomain [3]. This nuclear-capture event, in turn, drives actin polymerisation which is restricted to the juxtanuclear region [4] and LIMK-driven phosphorylation of cofilin [5], and both events are dependent on the RhoG GTPase.

Phosphorylation of cofilin opposes nuclear import of cofilin-actin, leading to depletion of G-actin from the nucleus [6] which, in turn, activates transcription of MRTF/SRF-target genes [7] to implement cell scattering and invasion.

\section{Supplementary Files}

This is a list of supplementary files associated with this preprint. Click to download.

- Movie1.mov 
- Movie2.mov

- Movie3.mov

- Movie4.mov

- Movie5.mov

- Movie6.mov

- Movie7.mov

- Marcoetal.Spreadsheet1.xIsx

- Marcoetal.Spreadsheet2.xlsx

- FigureS1.jpg

- FigureS2.jpg

- FigureS3.jpg

- FigureS4.jpg

- FigureS5.jpg 